\title{
A taxonomic synopsis of Altingiaceae with nine new combinations
}

\author{
Stefanie M. Ickert-Bond', Jun Wen² \\ I UA Museum of the North Herbarium (ALA), Department of Biology and Wildlife, University of Alaska \\ Fairbanks, 907 Yukon Drive, Fairbanks, Alaska 99775-6590 USA 2 Department of Botany, MRC-166, \\ Smithsonian Institution, P.O. Box 37012, Washington, D.C. 20013-7012 USA \\ Corresponding author: Stefanie M. Ickert-Bond (smickertbond@alaska.edu)
}

Academic editor: D. Soltis | Received 15 September 2013 | Accepted 27 November 2013 | Published 17 December 2013

Citation: Ickert-Bond SM, Wen J (2013) A taxonomic synopsis of Altingiaceae with nine new combinations. PhytoKeys 31: 21-61. doi: 10.3897/phytokeys.31.6251

\begin{abstract}
A taxonomic synopsis of the Altingiaceae is presented, including the taxonomic enumeration and distribution of 15 recognized species based on studies of 1,500 specimens from 24 herbaria throughout the distributional range of the taxa. Previous phylogenetic analyses based on several molecular markers have shown that Altingia and Semiliquidambar are nested within Liquidambar. All Altingia and Semiliquidambar species are now formally transferred to Liquidambar, which has the nomenclatural priority. The following nine new combinations are herein made: Liquidambar cambodiana (Lecomte) Ickert-Bond \& J. Wen, L. caudata (H. T. Chang) Ickert-Bond \& J. Wen, L. chingii (Metcalf) Ickert-Bond \& J. Wen, L. gracilipes (Hemsl.) Ickert-Bond \& J. Wen, L. multinervis (Cheng) Ickert-Bond \& J. Wen, L. obovata (Merrill \& Chun) Ickert-Bond \& J. Wen, L. poilanei (Tardieu) Ickert-Bond \& J. Wen, L. siamensis (Craib) Ickert-Bond \& J. Wen, and L. yunnanensis (Rehder \& Wilson) Ickert-Bond \& J. Wen.
\end{abstract}

\section{Keywords}

Altingia, Altingiaceae, Liquidambar, Semiliquidambar, taxonomic synopsis

\section{Introduction}

The Altingiaceae (the sweet-gum family) are a small family of trees that have been traditionally classified into members with a predominantly temperate distribution (Liquidambar L.) and those with a largely tropical to subtropical distribution (Altingia Noronha, Semiliquidambar H. T. Chang). The family is valued worldwide 
for its timber and fragrant resin (styrax) and is locally highly prized for the roots and bark used in traditional Chinese medicine (Vink 1957; Zhang et al. 2003; Ickert-Bond et al. 2007). Most noteworthy to biologists, Altingiaceae show a fascinating intercontinental disjunction in temperate regions of North America, W Asia and some higher elevational montane areas in subtropical Asia and Mexico (Ickert-Bond and Wen 2006). Furthermore, deep molecular divergence coupled with a high level of morphological similarity suggests a conserved morphology of some species, i.e., morphological stasis, an evolutionary phenomenon that has been proposed for many animal groups as well as some plants (Ickert-Bond and Wen 2006). Other members of the Altingiaceae exhibit morphological divergence in response to habitat diversity in the subtropics of eastern Asia. One member of the sweet-gum family, Semiliquidambar, has puzzled scientists since its discovery in the 1960s, due to its rarity and morphological intermediacy between the other two genera (Ferguson 1989).

Generally, the Altingiaceae were considered closely related to the Hamamelidaceae (see below). The family is recognized by the solitary capitate woody infructescences with many bicarpellate fruits, and male inflorescences in heads aggregating into racemes. Other systematic characters that differentiate the two families are less well known and understood. It is now generally agreed that the Altingiaceae are a distinct family (Magallón et al. 1999); for detailed comparisons see our other contributions (APG II 2003; Pigg et al. 2004; Ickert-Bond et al. 2005, 2007; Ickert-Bond and Wen 2006; APG III 2009).

The family name Altingiaceae is based on Altingia, first named by Noronha (1790) in honor of the former General Governor Alting of the East Indian colonies of the Netherlands (Hayne 1830). The family was formally designated in 1843 by Horaninow (Hoogland and Reveal 2005). The scientific name for Liquidambar L. is a combination of the Latin and Arabic words Liquidus and Amber meaning fragrant liquid or balsam (Yaltrik and Efe 2000). Most authors have recognized Altingia and Liquidambar to be members of the Hamamelidaceae s.l., most often at the subfamily level, while Endlicher in his Genera Plantarum (1840) segregated the Altingiaceae [s. Balsamifluae] from the Hamamelidaceae s. str and placed Altingiaceae in Juliflorae, between Platanaceae and Salicaceae, while the Hamamelidaceae were placed in Discanthae, between Loranthaceae and Bruniaceae. Subfamily Altingioideae was recognized by J. Williams in his revision of Balfour's Manual of Botany in 1855 . Bentham and Hooker (1883) treated Liquidambar and Altingia as distinct genera alongside other typical genera of Hamamelidaceae, without mention of subfamilies, but they recognized two categories (Abteilungen): (1) with the ovary containing 2- many ovaries, and (2) with the ovary containing a single ovary. Reinsch (1890) retained the traditional one family concept, but departed from the general consensus by splitting the Hamamelidaceae based on morphological and anatomical characters, that he considered to be more fundamental than the fruit, into three subfamilies: (1) Altingioideae including Altingia and Liquidambar and (2) Bucklandioideae including Exbucklandia R.W. Brown and Rhodoleia Champ. ex Hook., and (3) Hamamelidoideae including Corylopsis Siebold \& Zucc., Dicoryphe Thouars, Distylium Siebold \& Zucc., Eustigma Gardner \& Champ., Fothergilla L., Hamamelis 
L., Loropetalum R. Br., Parrotia C.A. Mey., Sycopsis Oliv. and Trichocladus Pers., (Semiliquidambar was not known at the time).

Baillon (1871) explicitly excluded Liquidambar and Altingia from the Hamamelidaceae, contrary to Bentham and Hooker, who included these two genera in the family. Baillon placed them in an intermediate position between Hamamelidaceae and Platanaceae. Traditionally, the Hamamelidaceae s.l. (including Altingiaceae) have been considered as a member of the Hamamelididae Takht. (Cronquist 1981; Takhtajan 1997). Recent molecular studies have shown this assemblage to be polyphyletic and support Altingiaceae and Hamamelidaceae s. str. as members of the saxifragoid clade within a larger rosid clade (Chase et al. 1993; Magallón et al. 1999; Soltis et al. 2000; APG II 2003; Fishbein and Soltis 2004; Soltis et al. 2007; APG III 2009; Soltis et al. 2011). Furthermore, relationships among some Saxifragales, and the remaining families (Altingiaceae, Cercidiphyllaceae, Daphniphyllaceae, Hamamelidaceae, and Paeoniaceae) also remain unclear (Feng et al. 1998; Qiu et al. 1998; Hoot et al. 1999; Savolainen et al. 2000; Fishbein et al. 2001; Soltis et al. 2000). In maximum likelihood analyses of a five-gene data set, Fishbein et al. (2001) recovered an optimal topology with Daphniphyllaceae and Hamamelidaceae sister to the remaining members of the clade; however, the precise branching order of these two early-diverging members of Saxifragales was unclear. Following Daphniphyllaceae and Hamamelidaceae, Altingiaceae, Cercidiphyllaceae, and Paeoniaceae appeared as successive sisters to a core clade of Saxifragaceae, Haloragaceae and Crassulaceae. Fishbein et al. (2001) also showed that the poor resolution obtained in Saxifragales is not due to violations of assumptions or to combining data partitions having conflicting histories or processes. Rather, their analyses suggest instead that the initial diversification of Saxifragales was indeed rapid. Within Saxifragales molecular phylogenetic results have rarely supported a sister relationship between Altingiaceae and Hamamelidaceae s. str. (e.g., Hoot et al. 1999; Fishbein et al. 2001, but see Fishbein and Soltis 2004). Most recently, based on over 50,000 bp Jian et al. (2008) have found strong support for a clade composed of the Paeoniaceae + woody clade (Cerdiphyllaceae, Daphnipyllaceae, and Hamelidaceae) Altingiaceae)) to be sister to the rest of the Saxifragales. The sister group relationship of Altingiaceae with Hamamelidaceae plus Cerdiphyllaceae and Daphniphyllaceae was also strongly supported in a supermatrix approach by Soltis et al. (2013).

Altingia and Liquidambar are each defined by several morphological characters and have been maintained as separate genera in modern taxonomic treatments (Vink 1957; Tardieu-Blot 1965; Zhang et al. 2003). Analyses based on several molecular markers suggest that Altingia is nested within Liquidambar (Shi et al. 1998; Shi et al. 2001; Ickert-Bond et al. 2005; Ickert-Bond and Wen 2006; Ickert-Bond et al. 2007, Wu et al. 2010) and that Semiliquidambar is of intergeneric hybrid origin between L. formosana-L. acalycina and $A$. obovata or A. chinensis. Yet our morphological analysis supports Altingia and Liquidambar as mutually exclusive sister clades (Ickert-Bond et al. 2005, 2007). The apparent incongruence of these phylogenies appears to be due to morphological convergence.

Characters that distinguish Liquidambar from Altingia are related to an open wind pollination syndrome and may represent convergences to temperate habitats, particu- 
larly, the presence of anthers borne on long filaments and the loss of stomium bifurcations would facilitate the wind dispersal of pollen (Hufford and Endress 1989), while long narrow styles on exserted fruits (Fig. 2D) may aid in the capture of pollen on the broad stigmatic surfaces in open habitats of temperate Liquidambar. Furthermore, additional synapomorphies for Liquidambar may also represent adaptations for a temperate distribution. These characters (elongate and tapered carpel shape, seeds with distal wings, and more tightly constructed infructescences) are related to seed rather than pollen dispersal. Several other families (e.g., Platanaceae) show a similar convergence among temperate members (Tiffney 1986; Crane 1989).

Character-state changes in Altingia seem to correlate with tropical and subtropical environments in eastern Asia and Indochina, whereas changes in Liquidambar correlate with temperate sites, where the genus is found today. Of the eight characters defining Altingia (Fig. 2A, B), four are reversals (characters 2-5: ratio of leaf length to width, leaf division, venation, and stipule size) (see fig. 86 of Ickert-Bond et al. 2007). The availability of diverse habitats in tropical and subtropical eastern Asia and Indochina facilitated the diversification of Altingia species in response to recent active uplifts of mountains in eastern Asia since the Tertiary (Morley 1999; Wen 1999, 2001; Ickert-Bond and Wen 2006).

To maintain the monophyly of the group in question (Potter and Freudenstein 2005), we place all taxa of Altingiaceae in Liquidambar (the earliest available name), and maintain the conserved name Altingiaceae for the family. Appropriate new combinations are provided below.

\section{Methods}

We evaluated all currently recognized taxa within Altingia, Liquidambar, and Semiliquidambar. Our study is based on: (1) field observations from throughout the distributional range of the taxa, with field visits to sites in Mexico (Veracruz), Vietnam, Cambodia, Indonesia and Guangdong, Hainan, Hong Kong, Hubei, Giangxi, and Zheijang provinces in China, and (2) the analysis of specimens in 24 herbaria (including available types): A, BK, BM, C, E, F, FI, FN, FUS, GH, HGAS, HN, IBSC, ISTO, K, LINN, LU, N, NFU, NY, P, PE, SYS, US. We previously provided detailed examination of the fruit anatomy and morphology (Pigg et al. 2004; Ickert-Bond et al. 2005; Ickert-Bond et al. 2006, 2007) and here provide an overview of some of the features that have been used to characterize the genera both for ovulate and staminate infrutescences. Measurements were made with an electronic caliper (Mitutoyo mod. CD-6"CS).

Pollen of selected species from all three genera were studied to assess the taxonomic utility in this group. Pollen samples were obtained from herbarium material deposited at $\mathrm{F}$ unless otherwise noted (L. chinensis [S. Ickert-Bond 1319]; L. excelsa [Widjaja s.n.], L. gracilipes [S. Ickert-Bond 1344], L. obovata [Wang 36153], L. poilanei [S. IckertBond 1296], L. siamensis [S. Ickert-Bond 1281], L. acalycina [Chui 3191 (MO)], L. formosana [C. Tan 93025 (2 sheets, MO)], L. styraciflua [Vazquez T. 153], L. chingii 
[S. Ickert-Bond 1330, S. Ickert-Bond 1320], and acetolyzed (Erdtman 1960), OTOTO coated (Kelley et al. 1973; Chissoe et al. 1994, 1995), freeze fractured (Skvarla et al. 1988), dried with HMDS (Nation 1983; Chissoe et al. 1994), mounted on stubs with double-sided tape, coated with approximately $200 \AA$ of gold in a Denton Vacuum Desk II vacuum evaporator or sputter coated with a gold/palladium target (60/40) in a Hummer VI Sputter Coating System (Chissoe and Skvarla 1996), and viewed with a JEOL JSM-880 scanning electron microscope (SEM) at 10-15 kV.

\section{Data resources}

The data underpinning the analyses reported in this paper are deposited at GBIF, the Global Biodiversity Information Facility, http://ipt.pensoft.net/ipt/resource. do?r=altingiaceae_synopsis.

\section{Taxonomic treatment}

Altingiaceae Horan., Osnov. Bot.: 271. 1841, nom. conserv. TYPE: Altingia Noronha, 1790. - Validated by a reference to an effectively, but not validly published Blume \& J. Fischer (Fl. Javae 17-18: 3. 1829, as Balsamifluae) family name with a description in Latin and proposed as an alternative name. - Isonyms: Horaninov, Tetracytys: 25. 1843 ("Altingiaceae (s. Balsamifluae"), validated by a reference to Blume \& J. Fischer (1829); see also Lindley, Veg. Kingdom: 253. 14-28 Mar 1846, validated by a description in English). The earlier Hayne (Flora 13: 172. 1830) name is a nom. nud.

Trees, deciduous or evergreen; terminal buds perulate, narrowly ovoid. Leaves petiolate; stipules usually present, linear, \pm adnate to base of petioles, caducous, leaving small scars; leaf blade palmately 3-7(or more)-lobed, or if entire lanceolate to ovate or obovate, leathery, discolorous, margin usually crenate-serrate, occasionally entire, venation pinnate or leaf blade palmately 3-7(or more)-lobed, venation actinodromous. Plants monoecious. Male inflorescence a globose to shortly cylindrical, pedunculate, many-flowered head, grouped in terminal or subterminal, compound racemes or panicles; each flower with 1-4 basal bracts. Female inflorescences capitate, subterminal or in lower part of male inflorescence, long-pedunculate, 5-30-flowered. Flowers unisexual. Sepals and petals absent. Male flowers: stamens (4-) many; filaments very short or absent; anthers obovate-ovoid, thecae 2-sporangiate, each dehiscing by a longitudinal slit or rudimentary valve, apex truncate; pollen spheroidal, polyporate. Female flowers: staminodes (also interpreted as carpellodes) absent or needlelike; ovary semi-inferior; ovules ca. 30-50 per locule, axile; styles subulate, divergent, often strongly recurved; stigmas papillose, basal parts or whole styles persistent in fruit. Infructescences globose, base truncate. Capsules woody, dehiscing loculicidally by two 2-lobed valves, also septicidally; staminode teeth and styles not persistent. Seeds many, upper ones sterile, one 


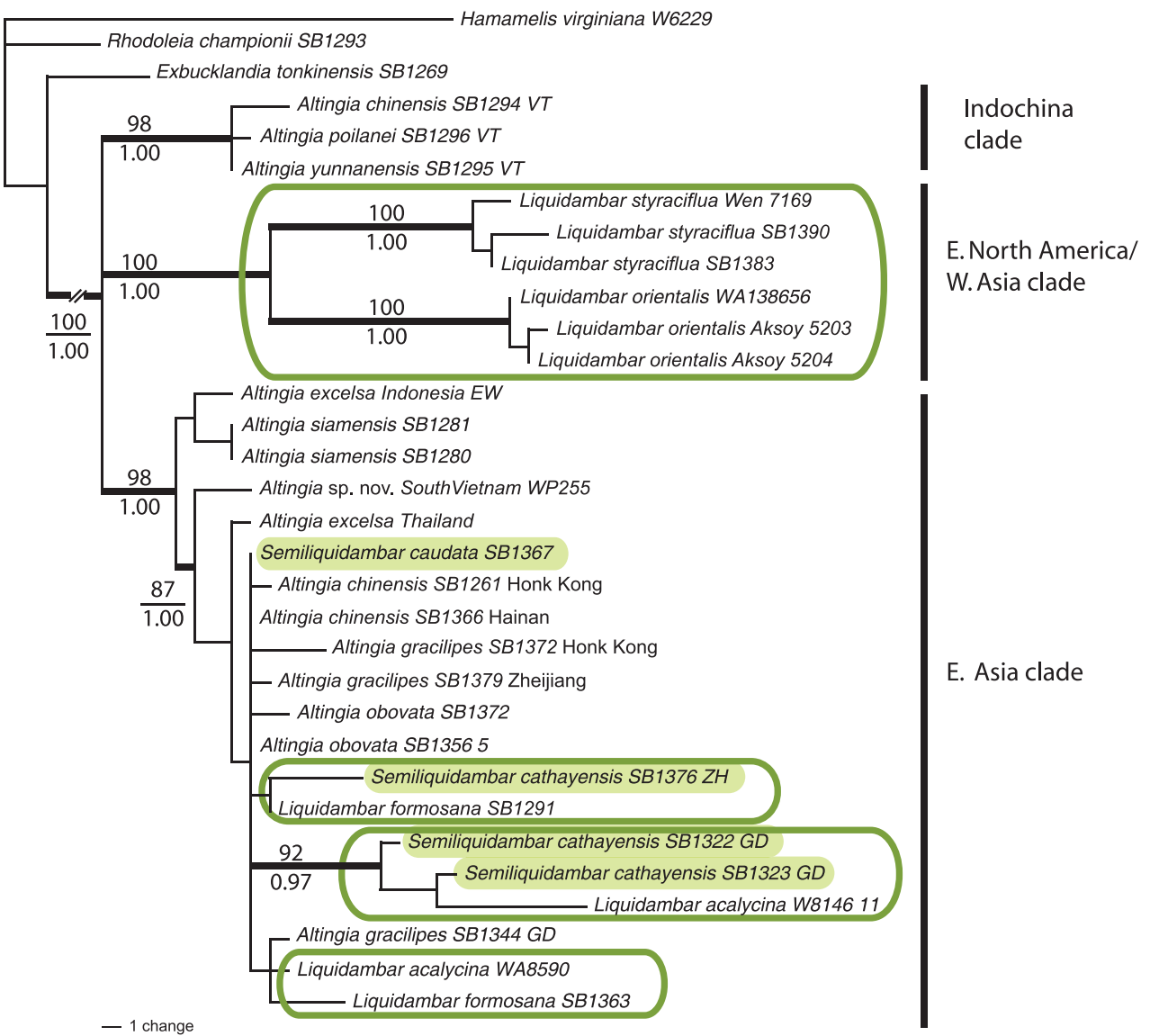

Figure I. Phylogenetic relationships of Altingiaceae based on maximum likelihood analyses of combined $c p$ DNA data. Phylogram is one of 14 trees $(-\operatorname{In} L=9927.72)$ derived from maximum likelihood analyses showing rates of substitution under K81uf+I model of substitution evolution (Modified from IckertBond and Wen 2006). Note: Break in branch lengths for the OG at left, and Semiliquidambar taxa with green shaded boxes, clades that include Liquidambar taxa have green outline boxes.

or a few lower ones fertile, flattened, narrowly winged along margin or only at apex; seed coat thick and hard; endosperm thin. $2 n=32$.

One genus and ca. 15 species: E, W, and SE Asia, Central, and North America.

Pollen morphology appears uniform throughout the family with spherical, pentaporate grains (Fig. 3A, E, I), that show scabrae and irregularly shaped perforations on the tectum (Fig. 3B-C, F-G, J-K) and a tectate-columellate exine (Fig. 3D, H, L; Ferguson 1989). Zavada and Dilcher (1989) found slight differences in the breadth of the columellae between Liquidambar styraciflua and Altingia obovata based on TEM imagery. Our analysis of the exine using freeze-fracturing and SEM shows slight difference of this characters between Liquidambar excelsa (= Altingia excelsa, Fig. 3D) 


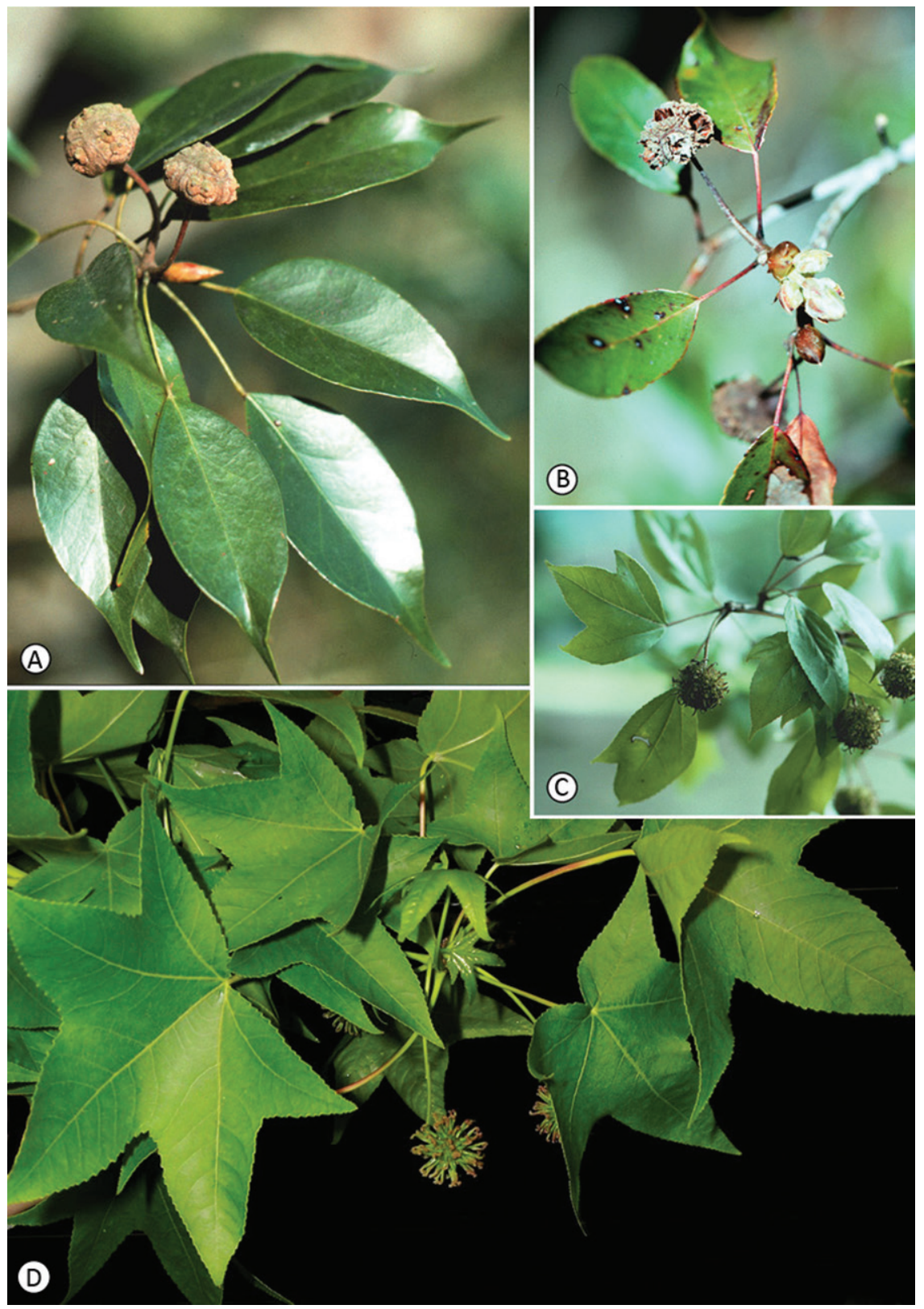

Figure 2. Morphological variation in Altingiaceae. A Liquidambar gracilipes with obconical flattened infrutescences and short styles. Leaves are simple, entire, choriaceous, and show a characteristic drip tip at the apex B Liquidambar siamensis also shows obconical flattened infructescences with simple leaves that have a serrate margin and lack of a drip tip $\mathbf{C}$ Liquidambar chingii showing variation in leaf morphology from palmately three-lobed leaves at left, to two-lobed leaves and simple leaves on the same branch. Infructescences are globose with long styles D Liquidambar styraciflua showing palmately five-lobed, chartaceous leaves, and globose infructescences with long styles. 

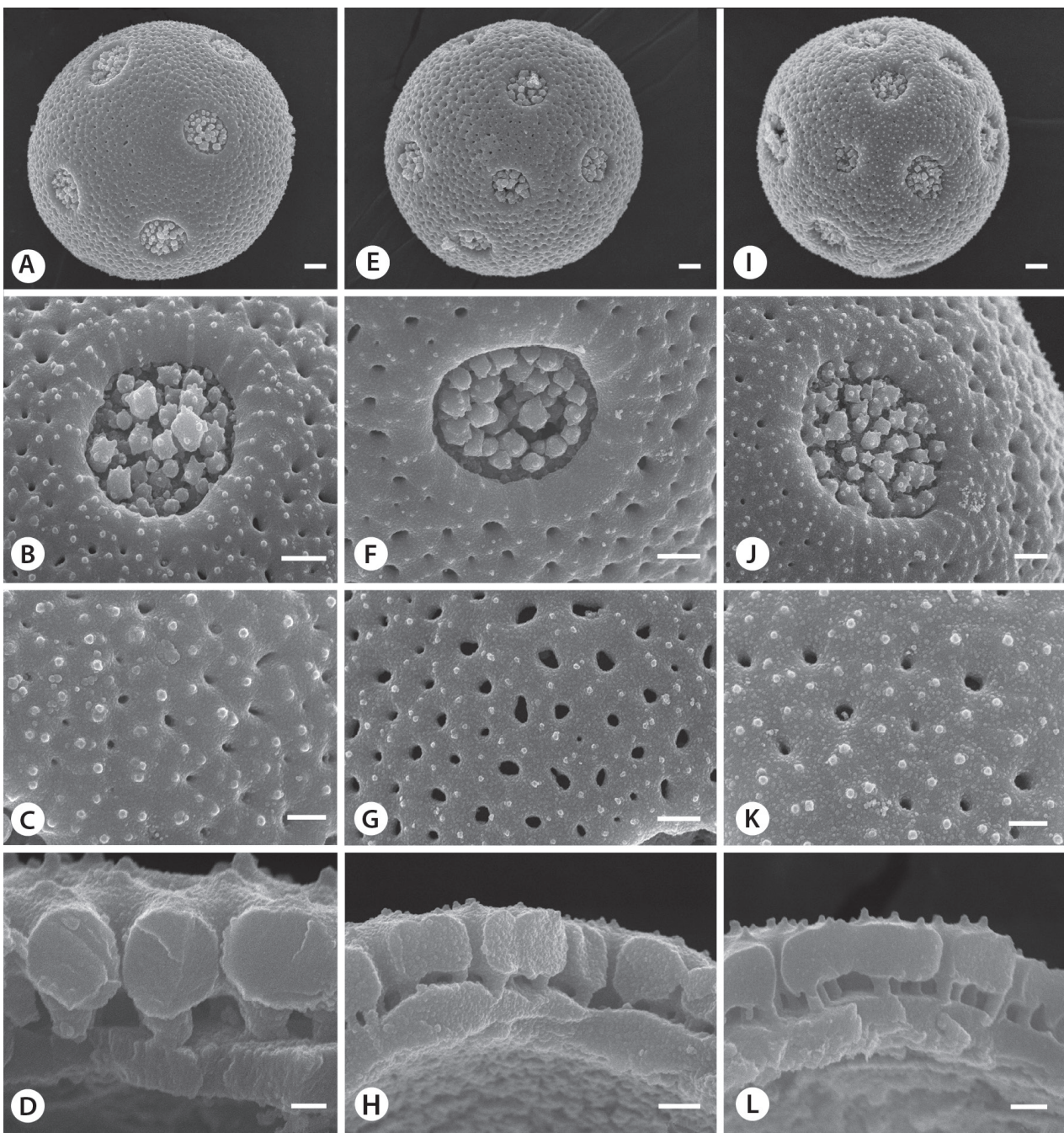

Figure 3. Pollen diversity in Altingiaceae. A-D Liquidambar excelsa E-H Liquidambar gracilipes I-L Liquidambar styraciflua A Pentaporate, spheroidal pollen grain B Details of pore showing the characteristic disintegration of the tectum $\mathbf{C}$ Details of the tectal surface displaying scabrae and irregularly-shaped perforations D Details of tectate-columellate exine, note columellae slightly thicker than those of comparable magnification in $\mathbf{H}$ and $\mathbf{E}$ Pentaporate, spheroidal pollen grain $\mathbf{F}$ Details of pore showing the characteristic disintegration of the tectum $\mathbf{G}$ Details of the tectal surface displaying scabrae and irregularly-shaped perforations $\mathbf{H}$ Details of tectate-columellate exine with thinner columellae than in $\mathbf{D}$ of Liquidambar excelsa I Pentaporate, spherical pollen grain J Details of circular pore showing eroded tectum in the pore proper $\mathbf{K}$ Details of tectal surface with irregularly-shaped perforations and scabrae $\mathbf{L}$ Details of the tectate-columellate exine with thin columellae as compared to Liquidambar excelsa in $\mathbf{D}$. Scale bars: A, E, $\mathbf{I}=2 \mu \mathrm{m}$, scale bars: $\mathbf{B}, \mathbf{F}, \mathbf{G}, \mathbf{J}=1 \mu \mathrm{m}$, scale bars: C, $\mathbf{H}, \mathbf{K}, \mathbf{L}=500 \mathrm{~nm}$, scale bar: $\mathbf{D}=200 \mathrm{~nm}$. 
and L. styraciflua (Fig. 3L), but the columellae in Liquidambar gracilipes (= A. gracilipes, Fig. $3 \mathrm{H}$ ) appear to be of equal width to those in the ones examined from L. styraciflua (Fig. 3L).

\section{Key to the species of Liquidambar s.l.*}

$1 \quad$ Leaves consistently palmately-lobed

2 Leaves of mature trees with three lobes, sometimes 5 in juvenile condition

3 Infructescence subglobose, with stout styles 4-6 mm long, curved; seeds with circular flange 1. L. acalycina

3' Infructescences globose, with fine styles 7-10 mm long, coiled; seeds with a terminal wing 7. L. formosana

2' Leaves of mature trees with more than 3, mostly 5 lobes, sometimes more than 5 lobes present

4 Infructescences with narrow style bases (up to $18 \mathrm{~mm}$ wide); areas between fruits appearing as a smooth rim Infrutescences with broad style bases (up to $30 \mathrm{~mm}$ wide); areas between adjacent fruits appearing braided 14. L. styracifua

1' Leaves variable, mostly unlobed or varying to lobed within a single branch

5 Leaves tri-nerved, mostly palmately-lobed, seldom unlobed

5' Leaves penninerved, strictly unlobed

6 Infructescences obconical with 5-8 fruits, base of the infructrescence with a prominent "skirt"

7 Leaves coriaceous, 2-3 cm wide, glossy above; margin entire, less often serrate; fruits up to $2 \mathrm{~cm}$ broad

8. L. gracilipes

7’ Leaves chartaceous, 3-4 cm wide, dull above; margin serrate; fruits broader than $3 \mathrm{~cm}$

13. L. siamensis

6' Infructescences mostly subglobose, with 10-30 fruits, base of the infructescence lacking a "skirt"

8 Leaves glossy above, margins distinctly revolute upon drying; endemic to Cambodia

2. L. cambodiana

8' Leaves dull above, margins not curled; of broader distribution in southeast Asia

9 Petioles $0.5-1.2 \mathrm{~cm}$ long

10 Leaves elliptical

4. L. chinensis

10' Leaves obovate

11 Leaf base acute, apex obtuse, lateral veins 8-10, conspicuous beneath 10. L. obovata

11'Leaf base subcordate to rounded, base acute, lateral veins 5-6, not prominent beneath. 12. L. poilanei 
9' Petioles $2-5 \mathrm{~cm}$ long

12 Leaves chartaceous...

12' Leaves coriaceous

13 Leaves 4-7 cm long, apex caudate, petioles slender, 1-1.5 mm thick; infructescences $1-2 \mathrm{~cm}$ broad 3. L. caudata

13' Leaves 8-13 cm long, apex acute, petioles stout, 2-3 $\mathrm{mm}$ thick; infructescences $2-3 \mathrm{~cm}$ broad. 15. L. yunnanensis

* Liquidambar multinervis is poorly understood at present and is not included in the key.

Liquidambar L., in Sp. Pl. 2:999. 1753.

http://species-id.net/wiki/Liquidambar

Altingia Noronha, in Verh. Bal. Gen. v. (1785) Art. ii. 9; ed. II. 41, nom. cons., syn. nov. Semiliquidambar H. T. Chang, in Acta Sct. Nat. Sunyatseni: 35, 1962, syn. nov.

Type. Liquidambar styraciflua L.

1. Liquidambar acalycina H. T. Chang, Acta Sci. Nat. Univ. Sunyatseni, 1959 (2): 33. 1959. TYPE. CHINA: Hunan: Wugang, H. T. Chang 4701 (holotype: IBSC 001053!). http://species-id.net/wiki/Liquidambar_acalycina

Distribution. China (Anhui, Guangdong, Guangxi, Guizhou, Hubei, Hunan, Jiangsu, Jiangxi, Sichuan, and Zheijiang). Representative specimens examined. CHINA: Fujian: Wuyi Mountain, Wuyi Shan Team 80-225 (MO); Wuyishan, Hengkeng, H.-Y. Zou 1984 (MO); Guangdong: Ruyuan Xian, C. Wang 44102 (MO); Guangxi: Damiaoshan, Shanfang Qu, Cidong Xian, Jiuwanshan, S.-Q. Chen 14715 (MO); Guizhou: Yinjiang Xian, along the trail between Zhangjiaba and Huguoshi on the west side of the Fanjing Shan mountain range, B. Bartholomew $1660(\mathrm{GH})$; Yinjiang Xian, vicinity of Xiapingsho on the west side of the Fanjing Shan mountain range, B. Bartholomew 1758 (GH, MO); Hubei: Lichuan, Metasequoia Region of Lichuan Xian (Hsien), vicinity of Lojiaba on the W side of the valley, B. Bartholomew $1950(\mathrm{GH})$; no locality, E.H. Wilson 513 (GH); vicinity of Shui-sa-pa, J.L. Gressitt 2415 (GH); Hunan: Henyuang, Goulowshan, J.B. Zuo 356 (MO); Hengshan, Nanyue, Longci, S.-Q. Chen 3346 (2 sheets, MO); Yizhang, Mangshan, Datangken, L.-H. Liu 542 (MO); Jiangxi: DeXin, De-xin county, K. Yao $11486(\mathrm{GH})$; Lushan, M.L. Nie 91192 (MO); Sichuan: S. Wushan, A. Henry 52181 (GH); Zheijiang: Linan Xian, Changhua, Bailongtanshan, P.L. Chin 961 (MO).

Cultivated. Arnold Arboretum, grown from seeds collected from Hubei province during the Sino-American Botanical Expedition (SABE $1950[\mathrm{GH}])$ near Lojiaba in the Metasequioa Valley of Lichuan Xian in October 1980. 
2. Liquidambar cambodiana (Lecomte) Ickert-Bond \& J. Wen, comb. nov. Basionym: Altingia cambodiana Lecomte, Bull. Mus. Hist. Nat. Paris 30: 391 (1924). TYPE. CAMBODIA: Mont d'Éléphant, Se mean phnom, Poilane 263 (holotype: P [P00749065!]; isotype: P [P003173561!]).

urn:Isid:ipni.org:names:77134706-1

http://species-id.net/wiki/Liquidambar_cambodiana

Note. A unique species with only three specimens from one locality in Cambodia known. The protologue does not include reference to where Poilane 263 is deposited. Furthermore, the two specimens at P are the only ones that include both "Poilane 263" and the locality information on the sheet (agreeing with the type description). The other two sheets, one at the Smithsonian (US 150518!) and one at the Edinburgh Botanic Gardens Herbarium (E 00181744!) include only labels that state Herb. Mus. Paris, Altingia cambodiana H. Lec. Institute Scientifique de Saigon - M. Poilane Reçu le 20 May 1921. These two later sheets (US 150518! And E 00181744!) most likely were part of the original material that Lecomte saw when he described $A$. cambodiana.

Distribution. SW Cambodia. Representative specimens examined. CAMBODIA. M. Poilane s.n. (US 150518!), (E 00181744!).

3. Liquidambar caudata (H. T. Chang) Ickert-Bond \& J. Wen, comb. nov. Basionym: Semiliquidambar caudata H. T. Chang, Acta Sci. Nat. Univ. Sunyatseni, 1962: 39. 1962. TYPE. CHINA: Fujian: Shaxian County, FUS expedition 53260 (holotype; FUS 47396- seen as photo!).

urn:Isid:ipni.org:names:77134707-1

http://species-id.net/wiki/Liquidambar_caudata

Semiliquidambar cuspidata H. T. Chang, Acta Sci. Nat. Univ. Sunyatseni, 1962: 39, 1962. TYPE. CHINA: Zhejiang: Jingning, Hangzhou Botanial Garden 7303 (holotype: PE [PE1392499!]; isotype: MO [MO4525923!].

Note. This taxon is similar to $A$. gracilipes, but its leaves are not strongly 3-nerved at the base.

Distribution. Fujian and Zhejiang provinces of China. Representative specimens cited. CHINA: Zhejiang: Ye Ling, Tai Shun County, Z.G. Mao 10237 (MO 4491051); Ying Chuan, Jing Ning county, S.Y. Chang 4837 (MO 4491052, MO 4536933).

4. Liquidambar chinensis Champ., Kew Journ. Bot. 4: 164, 1852. TYPE. CHINA: Hong Kong, Champion 325 (lectotype here designated: K [H2007/01764!]). http://species-id.net/wiki/Liquidambar_chinensis

Altingia chinensis (Champ.) Oliver ex Hance, in J. Linn. Soc. 13: 103, 1873. 
Altingia chinensis f. pubescens X.H. Song, in J. Nanjing Inst. Forest 4: 49, 1984. TYPE: CHINA: Guizhou: Libo, X.H. Song 1348 (holoype: NFU!).

Note. No specimen was mentioned in the type description. Champion $325(\mathrm{~K})$ is the only Champion specimen of Altingia found by us so far. It bears a label with a handwritten identification Liquidambar sp. nov. by Champion (based on comparison with other holotype material described by Champion at K). This specimen is thus designated as the lectotype for Liquidambar chinensis Champ.

Distribution. China (Guangdong, Guangxi, Guizhou, Hainan, Hong Kong), Vietnam. Representative specimens examined. CHINA: Guangdong: Kwai Shan, Tsing-lo-kong village, Ho-yuen district., W.T. Tsang 28544 (A); Lin Fa Shan, Sam Hang Shek T'au Village, Hwei-yang District, W.T. Tsang 25942 (A, E); Naam Kwan Shan, Tsengshing District, W.T. Tsang 20218 (E, MO); Poon Yue district, C.O. Levine 3158 (MO); Xinyi Xian, C. Wang 31828 (MO); Guangxi: Chen Pien District, S.P. Ko 56024 (A); Foo Lung, Sup Man Ta Shan, H.Y. Liang 69714 (A); Pingnan Xian, C. Wang 39334 (MO); Shap Man Taai Shan, near Hoh Lung village, SE of Shang-ze, Guangdong border (Shang-ze district), W.T. Tsang 22577 (A); Shap Man Taai Shan, near Iu Shan village, SE of Shang-ze, Guangdong border, Shangze district, W.T. Tsang 22189 (F); She-Feng Dar Shan, S. Nanning, R.-C. Ching 7937 (A); Tong Shan (along Guangdong border), near Sap-luk Po village (Waitsap district), W.T. Tsang 22788 (A); Hong Kong: Aberdeen Rd., H.C. Tang 590 (HK), Y.S. Lau 206 (HK); Jardin Botanique, E. Bodinier 1042 (E); Hong Kong Botanical Garden, S. Ickert-Bond 1274 (F); Lokchong, C.L. Tso 21049 (F); Luk Keng, Shek Pan Tam, P.-S. Choi s.n. (HK); Ma On Shan, K.E. Wong s.n. (HK); Shing Mun Country Park, Shing Mun Arboretum, S. Ickert-Bond 1261 (F); Zhejiang: Feng Yang Mtn., H.-Y. Zou 307 (A), H.-Y. Zou 761 (MO). VIETNAM: Lao Cai: SaPa, A. Petelot 2332 (MO); M. Brillet 19 (P, 2 sheets).

5. Liquidambar chingii (Metcalf) Ickert-Bond \& J. Wen, comb. nov. Basionym: Altingia chingii Metcalf, Lingnan Sc. Journ. 10: 413, 1931. TYPE. CHINA: Fujian: near Zhejiang border, $2800 \mathrm{ft}$., $R_{\text {. }}-\boldsymbol{C}$. Ching 2244 (holotype: SYS [SYS53818!]; isotypes: A [A 00043389!], IBSC [IBSC 001070!), NY [NY 00356121!], PE [PE 217977!]), SYS [SYS000957151!]).

urn:Isid:ipni.org:names:77134708-1

http://species-id.net/wiki/Liquidambar_chingii

Altingia chingii Metcalf var. parvifolia Chun, Sunyatsenia 1: 241, 1934. Semiliquidambar cathayensis H. T. Chang var. parvifolia (Chun) H. T. Chang, in Acta Sct. Nat. Sunyatseni: 38, 1962. TYPE: CHINA: Guangdong, Ying Tak, Wentong Shan, in mixed woods, H. Y. Liang 61283 (holotype: IBSC [IBSC001069!]; isotype: PE [PE 512712!]). Semiliquidambar cathayensis H. T. Chang, Acta Sunyatseni: 37, 1962. TYPE. CHINA: Guangdong: Ruyuan, S.P. Ko 53448 (holotype: IBSC [IBSC001068!]; isotype: PE [PE 509073!]. 
Semiliquidambar cathayensis $\mathrm{H}$. T. Chang var. fukienensis $\mathrm{H}$. T. Chang, in Acta Sct. Nat. Sunyatseni: 38, 1962. TYPE. CHINA: Fujian: Zhangping, Y. Ling 4522 (holotype: PE [PE419004!]; isotype: PE [PE83570!].

Semiliquidambar chingii (Metcalf) H. T. Chang, in Acta Sct. Nat. Sunyatseni: 38, 1962. Semiliquidambar chingii (Metcalf) H. T. Chang var. longipes Y. K. Li \& X. M. Wang, in Acta Botanica Yunnanica 8: 275,1962. TYPE. CHINA: Guizhou: Lipo Xian, Wujiahe, Exped. of Guizhou Academy of Science 76536 (holotype: HGAS!; isotype: SYS [SYS00072758!]).

Semiliquidambar coriacea H. T. Chang, in Acta Sct. Nat. Sunyatseni: 39, 1962. TYPE. CHINA: Guandong: Ruyuan, C. Wang et C.Y. Li 44074 (holotype: SYS [SYS123416!]; isotype: IBSC [IBSC001071!], PE [PE723787!]).

Distribution. China (Fujian, Guangdong, Guangxi, Guizhou, Jiangxi) and Vietnam (Ha Giang, Lao Cai). Representative specimens examined. CHINA: Guangdong: Da Ling Tan, Shan Shuai, Li Shan, Peixiang Tan 58681 (A); Hua Nan Agriculture University, specimen plant garden, Guangzhou, Zhiming Wu 85062 (MO); Nanling National Forest Park, S. Ickert-Bond 1307 (F), 1308 (F), 1310 (F), 1311 (F); Zhong Ba Shui, Zhong Ba, Yao Zu county, L. Deng 6681 (MO); Hainan: Bai Shui Ling, Dali County, Dali Mountains, Chengpo district, Qiungzhou County, L. Deng 3549 (MO); Qiongshong, Ying Ge Ling, eastern slope, National Geographic Society Hainan Expedition 46 (MO). VIETNAM: Ha Giang: Vi-Xuyen District, Minh-Tan community, H. Ngoc Kinh 169 (HN).

6. Liquidambar excelsa (Noronha) Oken, Allg. Naturgesch. iii. (3) 1539, 1841. Basionym: Altingia excelsa Noronha, Verh. Batav. Genootsch. Kunsten. 5(2): 9, 1790. http://species-id.net/wiki/Liquidambar_excelsa

Altingia caerulea Poir., Encycl. [J. Lamarck \& al.] Suppl. 5: 545, 1819.

Liquidambar rasamala Blume, Cat. Gew. Buitenz. 6, 1823.

Liquidambar altingiana Blume, in Flora Javae 17: 8, tt. 1,2, 1829.

Liquidambar cerasifolia (Wall. \& Griff.) Voigt, Hort. Suburb. Calcutt. 301. 1845.

Distribution. China, Bhutan, India, Indonesia (Java, Bali, Sumatra), Malaya, Myanmar, and Thailand. Representative specimens examined. BHUTAN. Sarbhang district: above Noonpani, $16 \mathrm{~km}$ along Sarbhang-Chirang road, A. Grieson 3581 (E). CHINA. Yunnan: between Muang Hing and Szemao and the Szemao hills proper, Southern Yunnan, J.F. Rock 2768 (GH); between Tengyueh and Lungling, J.F. Rock $7174(\mathrm{GH})$; no locality, G. Forrest 18414 (GH); Ping-pien Hsien, H.T. Tsai 61528 (GH); Shweli valley, G. Forrest 8763 (E, GH). INDIA. East Bengal, Griffith 3380 (A, GH, P); Jingale Bam near Nagahill, Prain $769(\mathrm{GH})$; Kachin Hills, Saden, Upper Burma, Mokim, Shaik s.n.; Ind. Or., Griffith 286 (GH); Ceylon, Royal Botanic Gardens, Peradeniya, sect. C 276, D.M.A. Jayaweera 1617 (GH). INDONESIA. Bali, Dedugul, Dumat 414 (MO), Bali Timur, Tabana. 2 km W of Candi Kuning, in natural areas of Kebun Raya, beyond introduced Altingia forest, 
McDonald \& Ismail 4966 (E, GH); Dutch West Indies, van de Koppel 3299 (MO); East Timor, Koepang, De Voogd 1772 (A); Java, Field Museum 373260 (A); Java, Ijoboshan, C.S. Sargen s.n.; W. Java, Nirmala Estate, gu Halimum area, Blukar and remnant of forest, M.J. v. Balgooy 2912 (GH); West-Java, Res. Batavia. Pasir Tjarewed, Land Boland, west of Bogor (Buitenzorg), elev. 600 m., Bakhuizen 6372 (MO); West Java, relict tree tp Tjibodas Mt. Garden, Gunung Gedeh (Mt.), Willem Meijer s.n. (MO); N. Sumatra, Karo plateau, Kaban Djahe, J.A. Loerzing 17368 (A); South East Java, H. O. Forbes 1201 (GH); Sumatra, Res. Benkaelen and Afd. Redjang, T.H. Endert 1068 (A); Sumatra, Sumatra's Westk. Moera-Laboch For. Serv, Neth. Ind. 18066 (A). MYANMAR. East of Paungdaw Power Station, west bank of the Paungdaw chaung river, J. Keenan 1407 (E), 1528 (MO); Patkai Mts., G. Schaap 13 (A); gorge of the Hkrang Hka, North Triangle (Hkinhum), F. Kingdon-Ward 20761 (A); south of Hpuginhku village, J. Keenan 3679 (E); Tenasserim Division, Tavoy District, J. Keenan 1940 (A). THAILAND. Nakhon Nayok, Khao Yai National Park, T. Smitinand 10848 (E); NE Kjonkaen, Phu Khieo, Game Reserve, ca. 80 $\mathrm{km}$ E of Phetchabun, Kyoto University 41655 (A).

7. Liquidambar formosana Hance, Ann. Sc. Nat. Ser. V. v. 215, 1866. TYPE. CHINA: Taiwan: Martio 1864, $R$. Oldham s.n. (lectotype here designated, P [P00749063!]; isolectotypes: NY [NY00356137!], P [P00749064!]).

http://species-id.net/wiki/Liquidambar_formosana

Liquidambar acerifolia Maxim., in Bull. Acad. Petersb. x. 486, 1866.

Liquidambar edentata Merrill, in Journ. Arnold Arb. viii. 6, 1927. (TYPE. CHINA:

Fujian [Fukien]: probably near Foochow, F.T. Metcalf \& T.C. Chang 877, fruit only

(Lectotype here designated: UC [UC 288168!]).

Liquidambar maximowiczii Miq., Ann. Mus. Bot. Lugd. Bat. 3: 200, 1867.

Liquidambar rosthornii Diels, Bot. Jahrb. Syst. 29(3-4): 380, 1900.

Liquidambar tonkinensis A. Cheval. in Bull. Econ. Indochine, n. s. 10: 839, 1918.

Note. A few specimens from Hunan and Guangdong exhibit pubescent stems, leaf surfaces and lobing attributable to young plants, previously annotated by Merrill (1934) as "L. formosana var. heterolobata": Tsang 20927 (MO, SYS), B. Xiong 8845, 9492, 10032 (MO), Hupeh Prov.: Wilson 513 (GH-2 sheets). This variety has never been formally published. These specimens are here referred to $L$. formosana.

The type of Liquidambar edentata Merrill is a mixture of a) L. formosana Hance [UC288168] as a single fruit mounted on the sheet, and b) a large branch of Acer tutcheri Duthie. Thus, lectotypification is required. One of the duplicates of F.T. Metcalf and T.C. Chang 877 at UC [UC258487] bears a label with the original handwriting of Merrill: "Liquidambar edentata Merr.", but the reference to the original publication ("Journ. Arnold Arbor. 8:6. 1927. Type in US Natl. Herb.") is not Merrill's own handwriting and was probably later added (as determined by John Boggan [US] from comparison with other holotype material at US described by Merrill). All duplicates of 
Metcalf \& Chang 877 [A 258487!, SYS 00072571!, UC 258487!] consist only of the branch of Acer tutcheri, without the Liquidambar fruit.

Distribution. China (Anhui, Chongqing, Fujian, Gansu, Guangdong, Guangxi, Guizhou, Hainan, Hong Kong, Hunan, Hubei, Jiangsu, Jiangxi, Shanxi, Sichuan, Taiwan, and Zheijiang), Laos, and Vietnam. Representative specimens examined. CHINA: Anhui: K. Ping 1597 (MO); Chien Shan Hsien, Tien Chu Shan, Chien Shan Hsien, C. S. Fan 280 (GH); Chiuhwashan, S.C. Sun 1302 (GH); Wang Shilong. Hefei Shi, Zushan, east slope, H.L. Yin 2031 (MO); Hwa Shan, C.S. Fan 75 (E); Chongqing (formerly in Sichuan Prov.): Chengkou Shi, T.L. Dai 103616 (MO); Nanchuan Hsien, W.P. Fang 811 (A); Fujian: Fan Hsioh Niao, Shaowu and vicinity, F.P. Metcalfe 9360 (GH); Hinghwa Dist., H.H. Chung 1006 (GH); Nanping Shi, Mangdangshan, G.-S. He 6179 (MO); Nanping Shi, 3800 Kan, G.-S. He 5677 (MO); Minhow Hsien: Pehling, near village dwellings, H.H. Chung $2132(\mathrm{GH})$; Sing-Shan, Foochow and vicinity, C.C. Tang 4655 (MO); Yenping: Buong Kang, H.H. Chung 3589 (GH); Yenping: Cha-ping, on slope, H.H. Chung 2901 (GH); Gansu: Wan Xian, Bikou, Z.-Y. Zhang 14271 (MO); Wan Xian, Motianling Shan, Baishui Jiang Nature Reserve, ENE of city of Bikou, D.E. Boufford et al. 37528 (MO); Guangdong: Chong Uen Shan near Kau Fung, Loh Ch'ang District, W. Tsang 20927 (A, MO); Guangdong, Canton and vicinity, C.O. Levine 1731 (MO); Lok F'au Mt., C.O. Levine 1572 (MO); Lung T'au Shan, Iu village and Yeung uk village, Lignan Team 12347 (MO); Nanling National Forest Park, S. Ickert-Bond 1305 (F); Nanling National Forest Park, S. Ickert-Bond 1305 (F), S. Ickert-Bond 1309 (F); Nanxiong, L. Deng 6602 (MO); Near Ninling city, on hill side, S. Ickert-Bond 1321 (F); Road to Jiangxi, along river, ca. $5 \mathrm{~km} \mathrm{~S}$ of Shitang city, S. Ickert-Bond 1326 (F); Road to Hunan from Nine Peaks, ca. 7 km outside of town, S. Ickert-Bond 1324 (F); Wan Tong Shan, Ying Tak district, T.M. Tsui 417 (MO); Yang Shan, and vicinity, South of Linchow, Yang Shan district, T.M. Tsui 516 (MO); T.M. Tsui 660 (2 sheets, MO); Xinyi Xian, C. Wang $31824(\mathrm{MO})$; Guangchow, White Cloud Hill on the way to the Temple, H.H. Chung 866 (GH); Guangxi: A.N. Steward $442(\mathrm{GH})$; Chuen Yuen, T.S. Tsoong 81972 (GH); Longjing, Daginshan, P.X. Tan 57605 (MO); Loh Hoh Tsuen, Ling Yun Hsien, A.N. Steward 29 (GH); Mts. Surrounding Pa Lau village, near Sui-Luk, SW o Nanning (Sui-luk village), W.T. Tsang $21817(\mathrm{GH})$; San-min village and vicinity, P'anku-shan and Ch'ao- t'ien-shan, Kwei-lin district, W.T. Tsang 28068 (GH); Ta Tseh Tsuen, A.N. Steward 1075 (GH); Guizhou: Jiangkou Xian, Baishuidong (white water cave) above the Minxiao River, SW of Jiangkou, B. Bartholomew 773 (GH); Lungli, $H$. Handel-Manzetti 185 (GH); Hainan: Bak Sa, S. K. Lau 25962 (GH). Bawangshanling, Z.-X. Li 3841 (MO); Chim Shan, Maan Ts'uen and vicinity, Ling Shui (Ling- tui) district, H. Fung 20245 (MO); Ka Chik Shan, Ka Chik Shan and vicinity, S.K. Lau 1645 (GH); Kam Kong, Yik Tsok Mau, Canton Christian College Herbarium 7700 (MO); Lingshui Xian, Nanqui, L. Deng 3116 (MO); Pak Shik Ling, Pak Shik Ling and vicinity, $\mathrm{Ku}$ Tung Village (Ching Mai district), C.I. Lei 355 (GH); Yangxin Xian, Longgang Zhen, C.-L. Ye 9689 (MO); Ya Xian, X.-R. Liang 62343 (MO); Yonlin, Yaichow, F.C. How 20124 (MO); Hong Kong: Chung Chi College, S.Y. Hu \& K.H. Yung 46 (MO); Chinese University of Hong Kong campus, S.Y. Hu 20064 (MO); S. Y. Hu 20953 (GH, 
MO); Hau T’ong Shan, Fuk Lung Monastery, Sin-Fung District, Fung Shue, Y.M. Taam 779 (GH); Hong Kong University campus, H.C. Tang 1473 (GH); Shing Mun Country Park, at the crossroads of Lead Mine Pass and Main Dam, S. Ickert-Bond 1260 (F); Hubei: Changchow, White Cloud Hill on the way to the Temple, H.H. Chung 866 (GH); Chikungshan, border of the provinces of Hupeh and Honan, on the divide between the Yang-Tze and the Hwai-ho rivers, L.H. Bailey s.n. (GH); Hinghwa Dist., H.H. Chung 1006 (GH); Hupeh (W) Arnold Arboretum Expedition, E.H. Wilson 795 (E); Lin District, C.O. Levine 3302 (GH); A. Henry 5218 (GH); A. Henry $7630(\mathrm{GH})$; Lung T'au Shan, Iu village and Yeung uk village, Lignan $12347(\mathrm{MO})$; Western Hupeh, Feng Heang, E.H. Wilson $513(\mathrm{GH})$; W. Hupeh, E.H. Wilson $218(\mathrm{GH})$; Xinyi Xian, C. Wang 31824 (MO); Yang Shan and vicinity, South of Linchow, Yang Shan district, T.M. Tsui 660 (MO); Yenping, Cha-ping, H. H. Chung 2901 (GH); Hunan: Henyuang, Goulowshan, J.B. Zuo 356 (MO); Liuyuang Xian, Longfa Zhen, Shizhu Feng, B. Xiong 2922 (MO); P'ing T'ou Shan, T'ang Wan village, Yi Chang district, W.T. Tsang 23613 (GH); Yushun Xian, Zhengxi, X-G. Li 204950 (MO); Jiangsu: Changsu, T.Y. Cheo 1149 (MO); Haichow village, J. Hers 2264 (GH); Hua Shan, Nanking, W.R. Carles s.n. (E); Kinling, E.H. Wilson 1639 (GH); Liu Liu Shan, near Haichow, J. Hers 608 (GH); Mao Shan, Tanjang, Tso 1803 (GH); Nanking, S.S. Chien $1019(\mathrm{GH})$; Yun-TaiShan, northern headland of Liuhe, extending into Kou Linhong, Lianyungong Bay, SAYTBET 45208 (GH); Yun-Tai-Shan, Lian-yun-gang, NE of Jiangsu prov., K. Yao 8505 (MO); Yuntai, Zikiang Shan (Purple Mountain), N extension of Mao Shan; NE of Sun Yat-Sen Memorial and Tomb, SAYTBET 45272 (GH); Jiangxi: Chuen Yuen, T.S. Tsoong 81972 (GH); De-Xin county, K. Yao 11561 (GH); Gangmaiping Xiang Huangyangjie, B. Xiong 5706 (MO); ); 3 km from Julianshan Nature Preserve entrance, S. Ickert-Bond 1327 (F); Kinkiang, E.H. Wilson $1628(\mathrm{GH})$; Oo Chi Shan, near Lam Uk Village, Lungan district, S.K. Lau 4809 (GH); San-min village and vicinity, P'an-kushan and Ch'ao-t'ien-shan, Kwei-lin district, W.T. Tsang 28068 (GH); Sang-su-ling, near Sih-cha-chieh Kan River, about 60 mi south of Nanchnag, Kinagsi, H.H. Chung 40 (GH); Shanxi: Yuyang Xian, Xiaoguojiaba, K.-J. Fu 5799 (MO); Sichuan: T. T. Yu 229 (GH, MO); Taiwan: Chiayi Hsien, Fanlu Hsiang, area nearby Pantienyen, Y.-R. Lin 516 (MO); Formosana Hokuto, A. Faurie $279(\mathrm{GH})$; Hsinchu Hsien, Wufengm Wushishan, S. Saito 8371 (MO); Kelung, O. Warburg 9810 (GH); Nanto, Province Nanto, E.H. Wilson 10031 (GH)Wuu Tsau street, T. Sozan 13490 (GH), L.L. Liu et al. s.n. (MO); Taipei, University campus, Y.R. Cheun s.n. (GH, MO); Tamsui, A. Henry 425 (GH); Taitum, U. Faurie 45 (GH); Zheijiang: Feng Yang Mountains, H.-Y. Zou 140 (GH); Kwangsi, Yung Hsien, Ta Tseh Tsuen, A.N. Steward 1075 (GH); Langquan, Taishui, R.C. Ching 4823 (GH); Lishui, Dagantou, S. Chang 6242 (MO); Sang-su-ling, near Sih-cha-chieh Kan River, about 60 li souht of Nanchnag, Kinagsi, H.H. Chung 40 (GH); Taichow, R.-C. Ching 1578 (GH), R.-C. Ching 4823 (GH); Tien Tai Shan, Kwohchingze, C.Y. Chiao 14238 (GH). LAOS: Bolikhamsay, Khamheut district, Ban Namphao, ca. $5 \mathrm{~km}$ east of town proper, D.D. Soejarto 11399 (GH); haut plateau, bassin d' attopen, Harmand 13007 (P). VIETNAM: Cao Bang: Ha Lang, municipality Thang Loi, vicinity of Thang Loi village, P.K. Loc et al. 1704 (MO); De Quang: Quang Tri, 
Lao Bao, M. Poilane 1317 (P); Bac Giang: Sau (Annam), Tonkin, foret de Pho-ve, $A$. Chevalier 2964 (P); Ha Tay: Da Chong, A. Petelot 5747 (A); Mt. Bavi National Park, close to Park headquarters, roadside, S. Ickert-Bond 1290 (F), Mt. Bavi National Park, roadside, S. Ickert-Bond 1291 (F); Hoa Binh: Kim Boi, T. Tien Phuong 2539 (HN); Lang Son: Dong Dang, B. Balansa 1156 (P); Huu Lien District, Huu Lsien Municipality, Huu Lien Protected Area, near village of Lan Cau, D.K. Harder et al. 4180 (MO); Lai moi sau cuoi, Savanne cay go, Chi lang, N. Tang Khoi 420, 421 (HN); Nghe An: Ke hhe, Donnat 38180 (P); Reserve forestier de Co - Ba (Vie-Nhe), F. Fleury 30170 (P); Ninh Binh: Cuc Phuong National Park, headquarters, east of helipad, N.M. Cuong 93 (GH, MO); Phu Tho: Foret de Dao gia, pres de Phu Tho, A. Chevalier 37471 (P); Than Hoa: Phong Y', M. Poilane 1610 (P); Tuyen Quang: Reserve forestiere de Niu-La, F. Fleury 37961 (F); Vinh Phuc: Ngoc Thanh, Me Linh, Phuong 4647 (2 sheets, HN).

\section{Liquidambar gracilipes (Hemsl.) Ickert-Bond \& J. Wen, comb. nov. Basionym: Altingia gracilipes Hemsl., in Hook. Ic. Pl. t. 2837, 1907. TYPE. CHINA: Fu- jian, S.T. Dunn Hb. Hongk. 2682. (Lectotype here designated HK [HK 10948!]; isolectotypes: A [A 00043390!], IBSC [IBSC 001001!]) \\ urn:lsid:ipni.org:names:77134709-1 \\ http://species-id.net/wiki/Liquidambar_gracilipes}

Altingi gracilipes Hemsl. var. serrulata Tutcher, Hong Kong Administrative Report 1914: M31. TYPE. CHINA, Hong Kong, Ukautang, W.J. Tutcher Herb. Hongkong No. 10947) (holotype: HK [HK 10949!, collected 25/5/1914]; isotypes: HK [Garden Department Hong Kong 1711!, collected 25/5/1914], HK [HK10950!])

Altinga gracilipes Hemsl. f. uniflora H. T. Chang, Sunyatsenia 7: 74: 1948. TYPE. CHINA, Fujian, Y. Ling 2295 (holotype: IBSC 001003!)

Distribution. China (Fujian, Guangdong, Hong Kong, and Zhejiang). Representative specimens examined. CHINA: Fujian: Jian Ou, Wang Mu Lin, Fujian Forestry Coll. 41 (MO); Long Yan, Jiang Shan, Shuang Che, H. Chen 1272 (F, MO); Nan Ping, Mang Tang Mountains, G. He 4690 (MO), G. He 5479 (MO), G. He 6155 (MO); Yong An, Hong Tian, Fujian Team 5567 (MO); Yungyung Mts., Fujian, central, S.T. Dunn 2682 (GH); Guangdong: Chaochow district, N.K. Chun 42718 (MO); Da Pu, Yan Shang, Min Ying, L. Deng 5438 (MO); Da Pu, Feng Xi, X. Wang 153 (MO); Da Pu, Ying Jian, Z. Li 963 (MO); Da Pu, Pu Cheng, X. Li 202545 (MO); Feng Xun, Da Tian, Bei Xi, X. Li 200921 (MO); He Yuan, Xin Feng Jian, B. Yu 103106 (MO); He Ping, Qing Zhou, Z. Wei 120579 (MO); Jiu Lian Xiang, Xiao Shui, Z. Wei 120217 (MO); Lian Ping, Zhong xin, Da Shui, Y. Liu 242 (MO); Ping Yuan, L. Dong 4331 (MO); Qiao Lin, Shi Hu, L. Dong 4685 (MO); Raoping Xian, N.K. Chun 42718 (2 sheets, MO); Rao Ping, Feng Huang, X. Li 200701 (MO); Rao Ping, Feng Huang Yu, H. Zeng 72101 (MO); Tung Koo Shan, Tapu district, Tan Shue, W.T. Tsang 21697 (GH, HK, P); Wu Hua, Shuang 
Hua, Z. Li 1162 (MO); Wu Hua, Qi Mu Zhang, Y.-J. Wang 56484 (MO); Xin Yi, Da Wu Lin, Z. Feng 36293 (MO); Yam Na Shan [Yit Nga Shan] Mei [Kaying] District, W.T. Tsang $21514(\mathrm{GH})$, Yunfu Xian, C. Wang $37057(\mathrm{MO})$; Hong Kong: M.T. Sin \& W.K. Woo s.n. (HK); Pat Sin Leng Country Park, Nam Chung trail, S. Ickert-Bond $1272(\mathrm{~F})$; Nam Chung, D. Lau 43 (GH, MO), T.W. Lau 98 (GH, MO), Y.-W. Lam 1429 (HK), K.-L. Yip 4082 (MO); Pat Sin Leng, Plover Cove Country Park, Plover Cove Reservoir, S. Ickert-Bond 1266 (F); Pat Sing Leng Nature Trails, L.T. Lo 629 (HK); Sha Tau Kok-luk Keng, S.Y. Hu 9984 (GH); Wu Kau Tan N.T., S.Y. Hu \& Y.C. Kong 175 (MO), Y.S. Lau 2634 (HK); Zhejiang: Between Ping Yung and Tai Suan, R.-C. Ching 2199 (E); Feng Yang Mtn., H.-Y. Zou 79 (MO), H.-Y. Zou 151 (GH), H.-Y. Zou $762(\mathrm{GH})$; Long Qian, Ju Shui, P.L. Chiu 1072 (MO); Qing Yuan, Wu Du Mountain, Z.G. Mao 10231 (MO); Shui Chang, Pu Nang, P.L. Chiu 1470 (MO); Taishun Hsien, Y.L. Keng $316(\mathrm{GH})$.

9. Liquidambar multinervis (Cheng) Ickert-Bond \& J. Wen, comb. nov. Basionym: Altingia multinervis Cheng, in Notes For. Inst. Nat. Centr. Univ. Nanking, Dendrol. Ser., No. 1, 3 1947. TYPE. CHINA: Guizhou: Chishui, Tiantaishan, 29 Oct 1938, P.C. Tsoong 256 (holotype, N seen as photo!; isotype, SYS 72729!). urn:Isid:ipni.org:names:77134710-1

http://species-id.net/wiki/Liquidambar_multinervis

Note. Specimens of L. multinervis show long-petioled papery leaves, with 10 lateral veins, and serrate margin, closely resembling L. siamensis or L. excelsa. Broken infructescences of the specimen at $\mathrm{N}$ suggest few fruits per infructescence, thus underscoring the closeness to L. siamensis, while Cheng (1947) described its close affinity with $L$. yunnanensis. This species is poorly understood, we have only seen the type collection that consists of a branch with multiple leaves and a crushed infructescence. The distributional discontinuity of L. multinervis in N Guizhou from those of L. siamensis and L. excelsa much further south may warrant specific status, but more material is needed. $\mathrm{N}$ Guizhou is not well explored botanically and with more exploration of this area, we might be able to better characterize this taxon in the future.

Distribution. China, N Guizhou.

10. Liquidambar obovata (Merrill \& Chun) Ickert-Bond \& J. Wen, comb. nov. Basionym: Altingia obovata Merrill \& Chun, in Sunyatsenia, 2: 238, 1935. TYPE. CHINA: Hainan: Yaichow, Ngai Yen, F.C. How 70369 (holotype: NY [NY 00356124!]; isotypes: A [A00043391!], HK [HK1713!], SYS [SYS 00095710!]). urn:Isid:ipni.org:names:77134711-1 http://species-id.net/wiki/Liquidambar_obovata

Distribution. China, Hainan. Representative specimens examined. CHINA: Hainan: no locality, H.Y. Liang 64371 (E), H.Y. Liang 64734 (GH), H. Y. Liang 62594 (P); 
C. Wang 35691 (MO); C. Wang 35897 (GH); Ding'an Xian, C. Wang 36153 (MO); Lingshui Xian, C. Wang 36638 (MO); Mo San Leng, N.K. Chun 44321 (GH); Qiong Zhong county, Cheng Po district, Da Li village (up?), Baishui Ling, Deng, Liang 3685 (MO); Waning County, Liulian Mts., Y. Zhong 4321 (MO); Waning county, Wumie district, Tongtie mountain (Ling), Z. Li 4972 (MO).

11. Liquidambar orientalis Mill., Gard. Dict. ed. 8, n. 2, 1768.

http://species-id.net/wiki/Liquidambar_orientalis

Liquidambar imberbis Ait., Hortus Kew. (W. Aiton) 3: 365, 1789.

Liquidambar orientalis Mill. var. integriloba Fiori, Ann. R. Inst. Sup. Agr. For. Naz. 9: 153, 1924.

Note. Liquidambar orientalis still needs lectotypification. The prologue states as follows: 'The seeds were sent by Mr. Peyssonel from the Levant, to the French king's garden at Marli, a few of which were sent to me by Mr. Richard, the king's gardener, which succeeded in the Chelsea [Physic] garden.'

When examining type material from FI of var. integriloba and the typical $L$. orientalis no clear distinction of lobing was observed. The FI specimens were identified as $L$. orientalis var. integriloba (A. Fiori $230-2$ sheets, A. Fiori $231-2$ sheets, G. Jannone s.n. -1 sheet, $A$. Fiori s.n. -1 sheet). When comparing material from Turkey (ISTO), the specimens collected by Aksoy 5202 (2 sheets) have margins that are sometime lobed beyond the typical 5 -lobing, also observed in Aksoy 5201 (ISTO-1 sheet), and Aksoy 5203 (ISTO - 4 sheets), while specimens identified as $L$. orientalis var. integriloba lack such lobing, as seen in Aksoy 5204 (ISTO-3 sheets) also from Turkey. This specimen (Murray 1020 - GH, $\mathrm{MO}$ ) lacks the typical lobing of var. orientalis and could thus be considered var. integrifolia.

Distribution. Southwestern Turkey and on the Greek island of Rhodes. Representative specimens examined. TURKEY: $\mathrm{C}_{2}$ Mugla: Kaycepra, Goner 9145 (MO); $\mathrm{C}_{2}$ Mugla: Kargi, $10 \mathrm{~km} \mathrm{~N}$ of Fethiye, G. Polunin 14923 (E); Mughla near Dogusbelen, Danish Bot. Trans-Asia Expedition III, No. 2081 (E); Mugla, between Köycefiz and Kavak Aksoy 5203 (ISTO); Mugla: Distr. Marmaris, Er. Koezcegiz, Khan 45 (E); Isparta, Sütçüler, Karacaören Aksoy 5204 (ISTO); Koezcegiz, J.S. Andersen 2081 (E); near Severagno, Khan et al. 45 (E); Paludal place (marsh), $1 \mathrm{~km}$ NE of Marmaris, E. Murray 1020 (A, E, MO); Vil. Mughla near Dogus belen, P.H. Davis 13474 (E). GREECE: collini a sud di Severagno, G. Jannone s.n. (FI); Peveragno, secus rivulum "Pelicano", K.H. Rechinger 8550 (E); convento d'Iskiati, A. Fiori 130 (FI), A. Fiori 230 (FI); era Alaeruna ed Apallaua, lengo il fiemme Saduras, A. Fiori 231 (FI); Rhodes Island, between Malona and Archangelos, K. Boratynska et al. 15 (K); between Malona and Archangelos, old trees along small stream, very frequent, $K$. Boratynska $164(\mathrm{~K})$; Salakos, hedges near stream, Davis 40317 (K); SE of Salakos, along stream, below orchard, K. Boratynska 15 (K). Cultivated: Italy: Rome, Martinetto s.n. (ASU). USA: Washington, University of Washington Arboretum, A.L. Bogle 1561 (ASU). 
12. Liquidambar poilanei (Tardieu) Ickert-Bond $\&$ J. Wen, comb. nov. Basionym: Altingia poilanei Tardieu, Fl. Camb., Laos \& Vietn., Fasc. 4, 95 (1965), in adnot. TYPE. VIETNAM: SaPa, M. Poilane 12844 (holotype, P 00317366!). urn:Isid:ipni.org:names:77134712-1

http://species-id.net/wiki/Liquidambar_poilanei

Distribution. Vietnam. Representative specimens examined: VIETNAM. Lao Cai: Sa Pa, China-Vietnam Team 8462 (HN); Ta Pinh Hmong village, some of the last remaining forest by small river across from rice paddies, S. Ickert-Bond 1296 (F).

13. Liquidambar siamensis (Craib) Ickert-Bond \& J. Wen, comb. nov. Basionym: Altingia siamensis Craib, in Kew Bull. 1928, 68. TYPE. THAILAND: Mŭang Pan, 700 m, evergreen forest, Doi Duan, Kerr 5110 (holotype: BK257949!). urn:Isid:ipni.org:names:77134713-1

http://species-id.net/wiki/Liquidambar_siamensis

Altingia tenuifolia Chun ex H.T. Chang, in Acta Sci. Nat. Univ. Sunyatseni, 1959(2): 34, 1959. TYPE. CHINA: Guizhou [Kweichow], Dushan, Y. Tsiang 6677 (holotype, IBSC!, isotypes: A [A00043392!], E [E00181734!], NY [00356122!]).

Altingia angustifolia H.T. Chang, Acta Sci. Nat. Univ. Sunyatseni, 1961 (4): 52, 1961.

TYPE. CHINA: Guangdong, Dapu, in dense forests, 10 Jun 1957, L. Deng 5031

(holotype: IBSC (IBSC 001000!); isotype: PE (PE00029853!).

Altingia takhtajanii Thai, Bot. J., URSS, 1. 996, 1965. TYPE. VIETNAM: Chieng-ve, Moc-chan, alt. 770 m, 13-III-1963, Thai Van Trung 108 (holotype: LE!)

Distribution. China (Guangdong, Yunnan), Cambodia, Laos, N Thailand, and Vietnam. Representative specimens examined. CAMBODIA. Forét de Phnom Penh, Komnhan, M. Bejaud 877 (P). Kampot: Bokor National Park, Pokopvil waterfall, near the head, S. Ickert-Bond 1280 (F), 1281 (F); Kampot, Bokor National Park, upper Popovill waterfall head, M. Monyrak 10 (A); INDONESIA. Java, Preanger Takoka, Koordes 15754 (P). LAOS: Fam Neva et M. Ham, M. Poilane 2000 (A, P); haut cours de la Zehepone entre A Chieng et Klem Zalo, M. Poilane 13500 (P); Pak Song, Sedone Prov., Sedone, J.E. Vidal 4461 (P). THAILAND. Nakorn Nayo: Khao Yai, Hardial 601 (A), J.F. Maxwell s.n. (MO); Nakhon Nayok, Khao Yai National Park, T. Smitinand 10848 (E); Nam Phnom, Prov. E., District Chaiyaphum, C.F. van Beusekom 4102 (MO). UNION OF MYANMAR: Tenasserim division, Tavoy district, east of Paungdaw Power Station, west bank of the Paungdae chaung, J. Keenan 1407 (E, MO). VIETNAM: semi flumen Da one in foret Bieu Loa, L. Pierre s.n. (P); Da Nang: Tourane, $100 \mathrm{~km}$ $\mathrm{S}$ of Hue, the later being Loureiro's type locality for the majority of the Cochinchina species, J. Clemens 3388 (A, MO, P); Gialai-Kontum: Dac Long, Dac Glai, Kontum, N. Kin Dao 182 (HN); Kbang, Kong Ha Nung, So Nglang, Xa Dong, Dac Glay, va mot so tinh Khac, no collector $745(\mathrm{HN})$; So Rang, An Khe, V. Xuan Phuong $586(\mathrm{HN})$; 
Khánh Hòa: Cay to hop, Nhatrang, M. Poilane 3228 (P); Hon Ba Mtns., Suoi Cat Village, D.D. Soejarto DDS 13561 (MO); Nui Chua National Park, Ninh Hai Distr., Vinh Hai Municipality, J.C. Regalado et al. HLF4449 (MO); Lam Dong: en peu au sud de la Mation agu cole de Blao pres du Haut Donai, M. Poilane 22153 (P); Loc Tan, Bao Lac, $N$. Tien Ban $469(\mathrm{HN}), H$. Tue 523 (HN). Ninh Thuan: Ka Rom pro: Phanrang, $M$. Poilane 9938 (2 sheets, P); Son La: Song Ma, N. Tien Ban 110 (HN).

14. Liquidambar styraciflua L., Sp. P1. 999, 1753. TYPE: Kalm, Herb. Linn. No. 1134.1 (LINN), (lectotype designated by Wijnands in Bot. Commelins: 109. 1983, [LINN HL1134.1, seen as image!]).

http://species-id.net/wiki/Liquidambar_styraciflua

Liquidambar barbata Stokes, Bot. Mat. Med. iv. 332, 1812.

Liquidambar gummifera Salisb., Prod. 393, 1796.

Liquidambar macrophylla Oerst., Am. Centr. 16 t. 10, 11, 1863. TYPE. NICARA-

GUA: Monte Pantasmo. A.S. Oersted 3050 (lectotype designated by Sosa in Flora

Veracruz 1:2. 1978 [C], as photo at MEXU; isolectotype [F!]).

Liquidambar styraciflua var. macrophylla (Oerst.) Nied. in Engl. \& Prantl, Nat. Pflanzenfam. 3, Abt. 2a: 124, fig. 69H, 1891.

Liquidambar styraciflua var. mexicana Oerst., Amer. Centr. 16, t. 11. 1863. TYPE. MEXICO: Veracruz: H. Bartholomé, F.M. Liebmann 3052 (lectotype designated by Sosa in Flora Veracruz 1:2. 1978 [C], as photo at ALA! and XAL).

Representative specimens examined. BELIZE: Cayo: Chiquibul, Ceibo Grande to Main Divide, A.K. Monro 2626 (MO), Ceibo Grande to drill sites track, M. Pena 1046 (MO); Chalate, Vicinity of La Palma, Dept. of Chalatenango, P.H. Allen 7265 (F). EL SALVADOR: Dept. Chalatenango: Along trails from San Ignacio to Las Pilas, west slope, J.M. Tucker 1209 (F); Chalate, El Jute, S. Calderon 1928 (F); Hacienda San Miguel near Metapan, M.C. Carlson 757, (F); On the road between San Salvador and La Palma, M.C. Carlson 608 (F); Salvador, Ana, forested slopes between Rio San Miguel and summit of Cerro El Pinal, Pine-aok zone, mountains near Finca San Jose, 10 km, N.C. Fassett 28302, (F, GH, MO); San Ignacio, La Palma, M. Hernandez 558 (MO); San Jose Igenio, P.N. Montecristo, V.M. Martinez 142, 454, (MO); $20 \mathrm{kms}$ al SO de Montecristo, R.A. Molina 12578 (F). GUATEMALA: Cerro Negro, J.A. Steyermark 51726 (A); Chicoyonito, Dept. Alta Verapaz, J.D. Smith 1855 (GH); Cocola, Reion de Cocola, northeast of Carcha, Dept. Alta, P.C. Standley 70309 (A); Dept. Alta Verapaz, Coban, H. Tuerckheim 1804 (E, GH, MO); Dept. Huehuetenango: Cerro Victoria, J.A. Steyermark 49713, (A); Dept. Zacapa: pine-covered slopes, Sierra de las Minas, J.A. Steyermark 29732 (A); Dept. Zacapa: along Rillito del Volcan de Monos, J.A. Steyermark 42409, (A); Huehne, Yalambohoch, E. Seler 3024 (GH); Nebaj, Dept. Quiche, A.F. Skutch 1739 (A); Sierra de las Minas, near San Geronimo, W.A. Kellerman 6412 (MO); Valley of Rio de las Violetas, north of Nebaj, G.R. Proctor 25212 (F, MO); Verapaz, Baja Verapaz. Mun. Chilasco, 6km al SW de Chilasco, 
L.P. Tenorio 14900 (MO). HONDURAS: Aldea El Carmelo, $1 \mathrm{~km} \mathrm{SE}$ of Valle de Angeles, G. Amador 172 (F); Floresta de pino-liquidambar de la Monatana Zanquin, R.A. Molina 2834 (F, GH); Lempira, Celaque National Park, ca. 7 km of Gracias, T.F. Daniel 9628 (MO); Rio Pijol Valley, 6-7 km south of Nueva Eperanza, R.L. Liesner 26609 (MO); Trencheras, $20 \mathrm{~km} \mathrm{~N}$ of Siguatapeque, $R$. Howard 627 (A); Valle Encantado, slopes of Mt. Uyuca, P.H. Allen 11181 (A); Dept. Morazan: entre Pena Blany, Lo de Ponce, L.O. Williams 17113 (GH); slopes of Cerro de Uyuca, P.C. Standley 887 (F); Dept. Altantida: vicinty of La Ceiba, near Danto river, T.G. Yuncker 8776 (GH); Dept. of Comayagua: edge of ravine near El Achote, T.G. Yuncker 5830 (F, GH); El Achote, near Siguatepeque, P.C. Standley 56158 (A); Comaya: bosque mixto y humedo de Barranco Trincheras, R.A. Molina 10807 (F); R. Selan, V. J. Rodriguez 2840 (F); vicinity of Siquatepeque, P.C. Standley 6497 (F); Cortes: Montana de Cusuco, Cordillera de Idalfonso, R.A. Molina 7254 (F); Montaña San Cristobal, sur de Agua Fria, R.A. Molina 7614 (F); Montaña Agua Fria, R.A. Molina 11342 (F); Montana San Idalfonso entre Banaderos y Cusuco, R.A. Molina 11454 (F). El Paraiso: Guinope, V.J. Rodriguez 1883 (F); Montaña Teupasenti, entre Junquillo y Teupasenti, R.A. Molina 11940, (F); Mt. Volcan, L. Williams 12190 (F); Paraiso; Lempira: Montana de Celaque, SE portion of massif, G. Davidse 34570 (MO); Mount Elaque National Park, J. Renfrow 16 (MO); Moraza: 20 km de Tegucigalpa, Montanña La Tigra, $A$. Rubio 63 (MO); Bosques del Volcan de Guaimaca, Cordillera Misoco, R.A. Molina 6127 (F); Campamento de Las Flores, P.C. Standley 13708 (F); Cerca de Montańa La Tigra, R.A. Molina 13755 (F); Faldas de Uyuca, R.A. Molina 983 (F); Los Planes. 25 km al N.E. de Tegucigalpa, S. Y. Chevez 119 (MO); Pinares entre La Piramide y Zambrano, R.A. Molina 11034 (F); Region of El Quebracho, P.C. Standley 23747 (F); Valle Encantado, slopes of Mt. Uyuca, P.H. Allen 11181 (F); Ocotep: Cordillera Merendon $10 \mathrm{~km}$ from Nueva Ocotepeque, R.A. Molina 22235 (F); Olanch: Montaña La Bellota en Cordillera Almendares, R.A. Molina 8430 (F); Siguate: $7.5 \mathrm{~km} \mathrm{SW}$ of Gracias, Lempira. Celaque National Park, T. Hawkins 176 (MO); Alrededor del Centro de Visitantes, D. Mejia 357 (MO); Guamil alrededor de Los Planes, D. Mejia 402 (MO). MEXICO: Chiapas: A $500 \mathrm{~m}$ al N de Rayon, camino de Pichucalco, S.E. Martinez 24118 (MO); 3 km northwest of Pueblo Nuevo Solistahuacan, R.F. Thorne 40034 (MO); at Rincon Chamula, $12 \mathrm{~km}$ northwest of Pueblo Nuevo, P.H. Raven 19784 (F); Colegio Linda Vista (Yerba Buena), G.L. Webster 17747 (MO); Colonia Kokijaz, A. Mendez Ton 6087 (MO); Mpio. De Bochil, Puliupul, C.H. Perino 3262 (MO); Ridge with Montane Rain Forest- Pine Oak, D.E. Breedlove 21762 (MO); San Andres Larrainzar, L.G. Gonzalez 233 (MO); Steep slope with montane rain forest, D.E. Breedlove $34365(\mathrm{MO})$; West of Tenejapa Center, D.E. Breedlove $6886(\mathrm{~F})$; Hidalgo: $5 \mathrm{kms}$ al oeste de Tianguistengo, M. Hernandez 5630 (MO), M. Hernandez 6912 (MO); $5 \mathrm{kms}$ al oeste de Tianguistengo, district Zacualtipan, H.E. Moore 1925 (GH); $4 \mathrm{kms}$ al oeste de Tianguistengo, (styraciflua) (MO); along Highway 85, D.H. Norris, 17397 (MO); district Jacala, municipality Chapulhuacan, H.E. Moore $2176(\mathrm{GH})$; district Molango, municipality Molango, H.E. Moore 1995 (GH); Nuevo Leon: Dulces Nombres, FG. Meyer 2799(MO); Oaxaca: $12 \mathrm{~km}$ al N de Guevea de Humboldt, distr. De Juchitan, R. Torres 2541 (F); Distrito Mixe: Municipio de Totontepec: Totontepec, Reyes Rivera, J. 257 (MO); Loma del Guayabo, Huautla de Jimenez, M. Hernandez 448 (MO); 
Municipio de Santiago Comaltepec: La Esperanza, R. Lopez Luna 27(MO); Municipio de Totontepec: Totontepec, R.J. Rivera $907(\mathrm{MO})$; Municipio de Totontepec, Chinantequilla, E. Vargaz Ruiz 44, 124 (MO); Puebla: Region Orizaba (second label states Xalapa), M. Bourgeau 2412 (GH); Tamaulipas: Rancho del Cielo (property of Frank Harrison), ca. 7 km WNW of Gomez Farias, W. Burger 26 (F); En el Rancho El Julilo, S.E. Martinez 3872 (F); Veracruz: $1 \mathrm{~km}$ above and NW of San Andres Tlalnehuayocan, M. Nee 26198 (F); 1 $\mathrm{km}$ al norte de Banderilla, Mun. Banderilla, J.I. Calzada 5246 (F); $1 \mathrm{~km}$ NW of Elotepec along (impassable), M. Nee 28898 (F); $2 \mathrm{~km}$ al NE de Banderilla, Rancho La Mesa, Banderilla, M.G. Zola 500 (F, MO); $3 \mathrm{~km} \mathrm{SSW}$ of Zongolica along gravel road to Chichiquila, Mun. Huatusco, M. Nee 29442, 29444 (F); 6 km (by road) ESE of Ixhuacan de Los Reyes, Mun. Ixhuacan de Los Reyes, M. Nee 22484 (F, GH); $10 \mathrm{~km}$ north of Huatusco in typical Liquidambar forest , G.K. Arp 4186 (F); 18 mi North of Jacala on Highway 85, D. Seigler 3601 (F); along Huayacocotla--Zontecomatlan rd., between Barro, La Calabaza and 5 km by road SE of Zilacatipan, Mun. Huayacocotla, M. Nee 26885 (F); along very winding road from Naolinco to Misantla, $13 \mathrm{~km}$ by road $S$ of turnoff to Yecuatla and $6 \mathrm{~km}$ by road $\mathrm{N}$ of Paz de Enriquez, Mun. Yecuatla, M. Nee 26393 (F); about $8 \mathrm{mi} \mathrm{N}$ of Teziutlan, toward El Mohon (near border of state of Puebla), E.W. Manning 53823 (GH); between Coscomatepec and Huatusco, F. Boutin 3480 (F); Camino Bastonal a Santa Marta, GomezPompa 5382 (F); Camino a Rancho Nuevo, Huayacotla, M. Hernandez M 1499 (F); Cerca de San Fernando, camino a San Fernando, O.R. Ortega 1234(F); Cerro de Macuiltepetl, Xalapa, M.G. Zola 697(F); Cerro de San Martin, J.I. Calzada 539 (F); Cerro de Villa Rica cerca de Mundo Nuevo, C.G. Castillo 1803, (F); Coacoatzintla, R. Ortega 837 (F); Consolapan, $4 \mathrm{~km}$ al norte de la desviacion, J.I. Calzada 5250 (F); en el volcan Santa Marta a $25 \mathrm{~km}$ al N de Catemaco, S.E. Martinez 3972 (MO); entre Zongolica y Nepopoalco, Zongolica, T.V. Vazquez 153 (F); gorge at Puente Acabaloya, ca. $1 \mathrm{~km}$ SE of Xico Viejo, $M$. Nee 26295 (F); ); Jalapa, C.G. Pringle 7754 (GH), C.L. Smith 1778 (GH); Jardin Botanico y Arboretum de Insitituto de Investigaciones Sobre Recursos Bioticos (INIREB), ca., $4 \mathrm{~km}$ SSW of Xalapa, H.H. Iltis 942 (F); kilometro 7, carretera San Adresito, Xalapa, M.G. Zola 616 (F); Lado SE de Laguna Catemaco, J.H. Beaman 5148 (F); Los Tuxtlas, Ocotal Grande, 5 km N de Mecayapan, Ibarra, G. Manriquez 2339 (MO); Mun. Juchique Ferrer La Cima, Plan de las Hayas, M. Hernandez 1610 (F); Mun. San Andres Tuxtla, Cerro Vigia al E de Volcan San Martin, J.H. Beaman 6276 (F); Mun. San Andres Tuxtla, cima del Volcan San Martin, J.H. Beaman 5970 (F); Mun. Xalapa, Jardin Botanico Clavijero, 3 km SE of Xalapa, M. Nee 29700 (F); Nacaxtla, Zongolica, T.V. Vazquez 260 (F); N Banderilla, J. Dorantes (MO); Bastonal-Sierra Santa Marta road, A. Gentry 32417 (MO); Near Highway 130 between Huachinango and Tulancingo, J. Conrad 3262 (F); Near Huatusco, F. Boutin 3494 (F); Nogales, E. Matuda 1157 (MO); Parque Ecologico of the Jardin Botanico Fco. Javier, Clavijero, 2km SW of Jalapa along road to Coatepec, M. Nee 23457 (F); Paz de Enriquez cloud forest, $15 \mathrm{~km}$ south of Misantla, L. Bohs 1798 (GH); Orizaba, Botteri 860 (GH); Paxopec, Municipio de Cacoatzintla, F.A. Ventura 19250 (MO); Rancho del Mesa, Municipio Banderilla, R.W. Marquez 997 (F); Rancho Nuevo entre Plan de las Hayas y Tierra, C.G. Castillo 1350 (F); Ridge on S side of gorge of Cascada de Texolo, 3 $\mathrm{km} \mathrm{SE}$ of Villa Xico (Xico=Jico), M. Nee 26005 (F); Road to microwave station from Ran- 
cho Chula Vista near Coscomatepec, F. Boutin 3485 (F); Sierra de Sta. Marta, M. Sousa 3548 (F, MO); Loc. Choapan, Mpio. Santiago Choapan, L.P. Tenorio 5329 (MO); Tenejapa, carretera Huatusco - Coscomatepec, R. Avendaño 277 (F); Tlalneuayocan, M.G. Zola 651 (F); Vaxin, cerca del Volcan San Martin, M. Sousa 3433 (F, MO); vicinity of small dam, 1/2 km W of Cinco Palos and $8 \mathrm{~km} \mathrm{NW}$ of Consolapan, Mun. Jalapa, M. Nee 29689 (F); Xonamanca, Zongolica, T.V. Vazquez 286 (F); Camino al Sumidero, Xalapa, M.G. Zola 746 (F). NICAGARAGUA: Jinotego: Esteli, N slope of Cerro El Fraile, W.D. Stevens 18095 (MO); Finca Aventina, in sierra east of Jinotega, P.C. Standley 10026 (F); Hacienda La Balestina, situada a unos $10 \mathrm{~km}$ E de la ciudad de Jinotega, A. Grijalva 250 (MO); Macizos de Penas Blancas, W.D. Stevens 11510 (MO); N slope of Volcan Yali, W.D. Stevens 15074 (MO; Madriz: 5 km SW of San Juan de Rio Coco, W.D. Stevens 17665 (MO); 5.2 $\mathrm{km} \mathrm{N}$ of San Fernando, W.D. Stevens (MO); Cerro El Fraile, P.P. Moreno 22766 (MO); Matagalpa: $5 \mathrm{~km}$ east of Yucul, C.E. Hughes 330 (MO); Cerca de entrada a bosque de Selva Negra, M. Araquistain 3538 (MO); El Ocotal km 134 between Matagalpa and Santa Maria, R.A. Molina 20435 (F, MO); Macizos de Penas, Blancas, SE side, W.D. Stevens 21054 (MO); Sta. Maria de Ostuma, Cordillera Central de Nicaragua, L.O. Williams 28004, 23388 (F); Wiwili: Deparmento de Nueva Segovia: ca. 5.2 km N of San Fernando, valley of Rio San Fernando, G.W. Stevens 3248 (MO); Jinotega, Municipio de Wiwili, Reserva Natural Kilambe, Communidad Aguas Frias, sector Caballo, R. Rueda 16311 (MO); Nueva Segovia: Cerro Mogoton, J.T. Atwood 9 (MO), D.A. Neil s.n. (F); Cerro Mogoton, $0.5 \mathrm{~km}$ W of El Volcan, $3.5 \mathrm{~km}$ NE of Dipilto, S. Tomlin 176 (MO); Dipilto, El Placer, km 247 carretera a Las Manos, P.P. Moreno 25910 (MO); Rio Arenal de Yali, Jalapa, J.T. Atwood 6816 (MO). U.S.A.: Alabama: Alabama Biological Survey, Auburn, Earle, F. S., (MO); Alabama, Rt. 11, near Knoxville, C.F. Reed 102737 (MO); Bienville Blvd., near Cadillac Sq., Dauphin Island, $R$. Deramus 1043 (MO); Rt. 21, 1 mi S of Riedmont, C.F. Reed 149022 (MO); Rt. 31, near Stapleton, C.F. Reed 103825 (MO); Arkansas: 0.8 mi north of Arkansas-Louisiana state line; Hulton, B.F. Bush 2425 (MO); Boston Mountains, P.H. Raven 26323 (MO); Bottoms, P.O. Malvern, Hot Springs Co., D. Delmaree 14502 (MO); Brazil, D. Delmaree 10971 (MO); Bruton, J.M. Greenman 4299, (MO); Flat woods, P.O. Star City, D. Delmaree 16748 (MO); Lake City, D. Delmaree 6945 (MO); Lookout Mountain. Near the line between Tennessee and Georgia which crosses the mountain, J.R. Churchill s.n. (MO); Monticello PO, D. Delmaree 1661 (MO); Near Nogo, G.M. Merrill 61 (MO); Nogo, G.M. Merrill 168 (MO); Ponca, L. Hubricht 1357 (MO); Rush, Marion County, along streams, E.J. Palmer 6031 (MO); Springs National Park, L.H. Pammel 189 (MO); Westfork, E.J. Palmer 8223 (MO); Woods along White River, near Calico Rock, E.J. Palmer 35546 (MO); Yell County: Bridge over the Petit Jean River, 2.6 miles north of Ola on State Hwy., T.G. Lammers 8304 (F); Conneticut: Driftway Lane, F.C. Seymour 20577 (MO); Woods, Tokeneke, near Darien, R. C. Ward s.n. (MO); Florida: Eglin Air Force Base; 11.7 mi east of Florida State Route 285 on Air Force Base Route 213, J.S. Miller 9554 (MO); Gainesville and vicinity of Lake Alice, T.B. Croat 25056 (MO); Near Jacksonville, A.H. Curtiss 4530 (MO); Hummock land, vicinity of Eustis, Lake County, G.V. Nash 860 (MO); Swales along Rt. 17, N of Yulee near Goodbread Circle, C.F. Reed 102948 (MO); Tall Timbers Research Station on S-12 north of 
Talahassee, D.A. Breil 376 (MO); Woods near Oak Hill, Volusia Co., C.F. Reed 35659 (MO); Woods, Rt. 41, 2 mi N of Brooksville, C.F. Reed 101308 (MO); Georgia: 1/2 mi S of Brewton, G.L. Bracewell 42 (MO); 2.4 mi E of Ailey on US 280, J.C. Solomon 2070 (MO); Alapha River swamp, 6 mi S of Stockton, J. Norsworthy (MO); Edge of Damp woods, Leslie, R.M. Harper 1391 (MO); Farm Woods, Rt. 301 just N of Ogeeche River, C.F. Reed $116746(\mathrm{MO})$; Flood plain and banks of Ty Creek, W.R. Faircloth $3374(\mathrm{MO})$; Hammock area (elevated terrain) surrounded by low pinelands on the E side of Moody AFB Reservation, W.R. Faircloth 3709 (MO); Illinois: $2.9 \mathrm{mi} \mathrm{S}$ of Gorham Rd. on ILL 3, then 3.9 mi E on Turkey Bayou Rd., bank of the big muddy river, J.C. Solomon 3719 (MO); Fountain Bluff, mesic woods along summit roadside, $R$. Carlson s.n. (MO); Horseshoe Island, G.N. Jones 12054 (MO); Mound city, E.J. Palmer 14826 (MO); Near Mississippi River, Chester, E.J. Palmer 44553 (MO); Tunnel Hill, E.J. Palmer 15227 (MO); Indiana: Awensvillle, C.F. Reed 3213 (MO); Near Arlington, C.F. Reed 3494 (MO); Brandywine, C.F. Reed 2840 (MO); Calvert Co., C.F. Reed 2463 (MO); Damp woods along River R. near Herald Beach, C.F. Reed 30915 (MO); David Hill Park, Balto, W.N. Lee 6 (MO); Double Rock Park, Parkerville, L.E. Schauer s.n. (MO); Flats Region, Guthrie Memorial Tract, R. C. Friesner 10161 (MO); Indian Head, C.F. Reed 3079 (MO); Lower Marlboro, C.F. Reed 4829 (MO); marches just south of Curtes Bay, Brandens Shore, C.F. Reed 31066 (MO); Middle River, C.F. Reed 3094 (MO); Oak-maple woods, Rt. 313, 1 mi N of Mossey, C.F. Reed 127985 (MO); on Rantan Formation, Elk Neck State Park at Rogus Harbor Boating facility cutoff, C.F. Reed 126623 (MO); Pt. Lookout .527, St. M., C.F. Reed 2476 (MO); Shiloh, C.F. Reed 409 (MO); Swampy woods E of Patiewment, S of Upper Marlboro, C.F. Reed 22817 (MO); Swampy woods near Pomonkey, C.F. Reed 23414 (MO); Wicomico, C.F. Reed 2533 (MO); woods along road to Southland Bog, C.F. Reed 125997 (MO); woods just north of East Riverside, C.F. Reed 29353 (MO); Woods Point Park, Bush R., C.F. Reed 128078 (MO); woods south of Drum Point, C.F. Reed 29290 (MO); Warwick, between Rt. 282 and Rt. 301, C.F. Reed 136283 (MO); Kentucky: 1 mile north of Cogswell, Rowan County, wet ditches, C.F. Reed s.n. (MO); 1 mile north of Cogswell, Rowan County, wet ditches, C.F. Reed 13437 (MO); along Ison Creek, on igneaous mica-perodotite dike west of Stephens, C.F. Reed 115816 (MO); along Axley Branck, Morehead, H. Williston 111 (MO); along Maxon Rd., just w of Concord, off Rt. 60, C.F. Reed 110706 (MO); Bowling Green, S.F. Price s.n. (MO); Georgetown, C.F. Reed 81477 (MO); Low woodlands, Rte. 68, near Erie, C.F. Reed 129641 (MO); Near Reed, Rt. 60, C.F. Reed 117576 (MO); Near Wago, Rt. 90.3 mi W of Albany, C.F. Reed 52888 (MO); Redd Hallow, L.B.L., 0.5 mi W of Kentucky 94, K. Hutchens 12 (MO); Rt. 42, Skylight, C.F. Reed 44907 (MO); Robey's Swamp, 3 mi NW of Franklin, C.F. Reed 82311 (MO); Rt. 45, 1.5 mi NE of Water Valley, C.F. Reed 52801 (MO); Rt. 470, Larue Co., near Buffalo, C.F. Reed 45066 (MO); Woods at Lovely, C.F. Reed 75623 (MO); Woods, Diohmans Springs, $7 \mathrm{mi}$ NW of Barbourville, C.F. Reed 7176 (MO); Woods near Goddard, Fleming Co., C.F. Reed 10446 (MO); Louisiana: 15 ft. from University Lake along Lousiana 42, S of Baton Rouge, K. Sijam s.n. (MO); 2 miles north of Gorum, Kisatchie Wood, Natchitiches Parish, J. Ewan 17630 (MO); 6 mi West of Ruston on Lousiana 507, P. Lohman 31 (MO); Cemetery, waste area and neighboring woodlands, J.K. Parrott 427 
(MO); Cypress Creek bottom, two miles N of Vienna, G. Fischer 20 (MO); just north of D’Arbonne Fire Tower, P. Both 315 (MO); Ponchatoula, Williams Lumber, (MO); Mississipi: Meramec State Park. Cambell Hollow, D. Dress 4 (MO); Natchez, F. Shimak s.n. (MO). Ocean Springs, Shekan, J., (MO); Missouri: at base of limestone bluffs alongs Frederick Pork near "The Narrows", west of Calm, J.A. Steyermark 18919 (MO); base of Black Mtn., J.A. Steyermark 21077 (MO); base of wooded lime slopes along spring branch of Williams spring on east side of current river, T25N, R1E, sect. 34, SW of Grandin, J.A. Steyermark 11853 (MO); Bilsted, Gum near Illmo, A. Christ s.n. (MO); Campbell Mts., B.F. Bush 227 (MO); Ca. 8 mi NE of Salem (T35 R4W S33 S2 SE4), Upper Ozark Section of Ozark Natural Division. Indian Trail conservation Area, T. Smith 3500 (MO); Columbia, near corner of Paquin an Waugh Streets, C. Dietrich 265 (MO); Kirkwood, $F$. Comte 437 (MO); low ground area in valley Blue Spring Itol, T27 N, R7 E sect. 20, 2 mi E of Chaonia, J.A. Steyermark 6313 (MO); lowland woods between Menfro and Belgique, 2 mi southwest of Belgique, J.A. Steyermark 14027 (MO); on a siding of the Terminal Railroad Association, V. Muehlenbach 3361 (MO); Pleasant Grove, B.F. Bush 258 (MO), K.K. Mackenzie 421 (MO); Poplar Bluffs, G. Letterman s.n. (MO), H. Eggert s.n. (MO); rich swampy woods on elevated portion, J.A. Steyermark 8662 (MO); Timber Tract, $10 \mathrm{mi}$ southwest of Wolf Island; Low woods along mud creek, T26N, R7E, sect. 20, 2 mi northwest of Rombauer, J.A. Steyermark 11294 (MO); Williamsville, E.J. Palmer 4805 (MO); Woods, Butler County, B.F. Bush 3721 (MO); North Carolina: Dry open woods east of Chapel Hill off 15-501, J.P. May 95 (MO); near Sisk's house, foot of Table rock, S.K. Small 292 (MO); Swamps, Bladen Country, Biltmore , 4178, (MO); Open marshy area, Durham, C. Henninger s.n. (MO); Woods just S of Grandy, Currituk Co., C.F. Reed 41634 (MO); Oklahoma: Broken Bow, E.J. Palmer 10496 (MO); growing on flat ground in valley, $H$. Newton $94(\mathrm{MO})$; near Page, G.W. Stevens $2634(\mathrm{MO})$; wet sandy loam soil in a wood, 4 mi SW of Broken Bow, R. Stratton 585 (MO); Pennsylvania: N of Boulevard, along Philadelphia-Trenton border of P.R.R., Cedar Grove, J.W. Adams 177 (MO); South Carolina: 1 mile north of Bloomfield, low pasture, beside Hwy 25, A. Chandler 2047 (MO); Anderson, J. Davis s.n. (MO); McKinney Spring, J. Davis s.n. (MO); Simpson's Mill near Anderson, J. Davis s.n. (MO); Upper coastal plain, sandy soil, M.L. Conrad 11171 (MO): Tennessee: Hancock-Claiborne County line, Damp cove, Vogenberger 8066 (MO); Knoxville, A. Ruth s.n. (MO); Aumville (?), A. Ruth 271 (MO); Nashville $12 \mathrm{mi}$ west on River Rd., E. Quaterman 1036 (MO); Near Memphis, E.J. Palmer 17516 (MO); Near Shepherd, E.J. Palmer 17478 (MO); Texas: 2nd Bottom, E.D. Marshall 8711 (MO); Big Thicket National Preserve. Lance Rosier Unit. Ca., J. Stone 3096 (MO); Houston, B.F. Bush 16, 22 (MO); Livingston, Polk county, E.J. Palmer 5235 (MO); West Virginia: Banks of James River, near Richmond, J.R. Churchill s.n. (MO); Camden Ave., near Ritchie St., Buchanon, G.B. Rossbach s.n. (MO); Carrollton, Isle of Wight Co, C.F. Reed 102408 (MO); Huntington, Williams 306 (MO); Miles Creek, Rt. 58, 6 mi S of South hill, C.F. Reed 53398 (MO); Paducah, McCracken County, E.J. Palmer 17884 (MO); Pigeon creek near Enons School, Mingo County, E.E. Berkley 966 (MO); Rt. 617 off Rt. 205, NE of Edgehill, C.F. Reed 130382 (MO); woods near Norfolk, F. Blanchard vicinity of Norfolk, M.C. Jensen s.n. (MO); Walls Run Creek at Rt. 10 near Rt. 609, C.F. Reed 89698 (MO); 
woods south of Suffolk, C.F. Reed 8946 (MO); s.n. (MO); woods, 8 mi SW of South Hill, Rt. 58, Mecklenburg Co., C.F. Reed 53404 (MO); woods along Rt. 14, Mattaponi, King and Queen Co., C.F. Reed 45994 (MO); woods just E of Pohick Church, on Old Colchester Rd., C.F. Reed 103177 (MO); woods just W of Camplain P.O., Rt. 17, C.F. Reed 100957 (MO).

15. Liquidambar yunnanensis (Rehder \& Wilson) Ickert-Bond \& J. Wen, comb. nov. Basionym: Altingia yunnanensis Rehder \& Wilson, in Sargent Pl. Wilson. I: 422 (1913). TYPE. CHINA: Yunnan: Mengtze, A. Henry 10395 (Holotype: A [A0043393!]; isotypes: K [H2007/01764!], NY [NY00356723!]). urn:Isid:ipni.org:names:77134714-1

http://species-id.net/wiki/Liquidambar_yunnanensis

Distribution. China (Yunnan), Vietnam. Representative specimens examined. China, Yunnan, Mengtze, A. Henry 11082 (A). Vietnam: Lao Cai: road to O'Qui Ho, SaPa, Institute Bot. Yunnanica s.n. (HN); Cao Bang: Cao Son, Poilane 19024 (P000317376!).

\section{Doubtful names}

1. Altingia indochinensis H. T. Chang, Acta Sci. Nat. Univ. Sunyatseni, 1961 (4): 53 (1961). Type: Indochina, without locality, M. Poilane 2000. We have not located the type specimen.

According to Chang (1961), this species is closely allied to A. gracilipes Hemsl. and its variety $A$. gracilipes Hemsl. var. serrulata Tutch., but differs from them by longer, oblanceolate or oblong leaves which are cuneate at the base, more robust petioles, and multiflorous heads.

\section{Excluded names}

1. [Araucariaceae Altingia cunninghamii in Hort. Brit. [Loud.] 403 (1830).

Notes: =Araucaria cunninghamii]

Altingia cunninghamii J. Ross, Hobart Town Almanack (1835) 66.

Remarks. Given as a name without description by G.Don in J.C. Loudon, Hort. Brit. (1830) 403. Not in Index Kewensis. "lately discovered by Mr. Ronald Gunn, at the falls of the river Meander and also by Mr. J.W. Scott, the collector on the banks of the Huon".

2. [Araucariaceae Altingia excelsa in Hort. Brit. [Loud.] 403 (1830).

Notes: =Araucaria excelsa] 


\section{Acknowledgements}

We would like to thank the curators of the following herbaria for sending specimens on loan or for providing access to collections: A, BK, BM, C, E, F, FI, FN, FUS, GH, HGAS, HN, IBSC, ISTO, K, LINN, LU, N, NFU, NY, P, PE, SYS, US; and Jim Reveal and John Boggan for helpful discussions on nomenclature.. This study was supported by a Boyd Postdoctoral Fellowship and a grant from the Field Dreams Program, Women's Board, the Field Museum of Natural History, National Geographic Society Explorers Grant NGS-7644-04, and a generous donation by the Wallace Desert Gardens, Scottsdale, Arizona, to S.M.I.-B.; and NSF DEB-0108536, Department of Botany and the Laboratory of Analytical Biology of the Smithsonian Institution, and a collaborative research grant from the Institute of Botany of the Chinese Academy of Sciences, to J.W.

\section{References}

APG II (2003) An update of the Angiosperm Phylogeny Group classification for the orders and families of flowering plants: APG II. Botanical Journal of the Linnean Society 141: 399-436. doi: 10.1046/j.1095-8339.2003.t01-1-00158.x

APG III (2009) An update of the Angiosperm Phylogeny Group classification for the orders and families of flowering plants: APG III. Botanical Journal of the Linnean Society 161: 105-121. doi: $10.1111 / j .1095-8339.2009 .00996 . x$

Baillon H (1871) Nouvelles notes sure les Hamélidées. Adansonia 10: 120-137.

Bentham G, Hooker JD (1883) Genera Plantarum. London: Lovell, Reeve and Company.

Chase MW, Soltis DE, Olmstead RG, Morgan D, Les DH, Mischler BR, Duvall MR, Price RA, Hillis HG, Qiu Y-L, Kron KA, Rettig JH, Conti E, Palmer JD, Manhart JR, Sytsma KJ, Michaels HJ, Kress WJ, Karol KG, Clark WD, Hedrèn M, Gaut BS, Jansen RK, Kim K-J, Wimpee CF, Smith JF, Furnie GR, Strauss SH, Xiang Q-Y, Plunkett GM, Soltis PS, Swensen SM, Williams SE, Gadek PA, Quinn CJ, Equiarte LE, Golenberg E, Learn GH Jr, Graham SW, Barrett SCH, Dayanandan S, Albert VA (1993) Phylogenetics of seed plants: an analysis of nucleotide sequences from the plastid gene $r b c \mathrm{~L}$. Annals of the Missouri Botanical Garden 80: 528-580. doi: 10.2307/2399846

Chang HT (1961) Notulae Plantarum Austro-Sinicarum. Acta scientiarum naturalium Universitatis Sunyatseni 1961 (4): 53.

Cheng WC (1947) New Chinese trees and shrubs. Research Notes, Forestry Institute, National Central University, Nanking, Dendrological Series 1:1-4.

Chissoe WF, Skvarla JJ (1996) Combining sputter coating with OTOTO treatment to eliminate charging artifacts in pollen preparations. Proceedings of the Oklahoma Academy of Science 76: 83-85.

Chissoe WF, Vezey EL, Skvarla JJ (1994) Hexamethyldisilazane as a drying agent for pollen scanning electron microscopy. Biotechnology and Histochemistry 69: 192-198. doi: $10.3109 / 10520299409106286$ 
Chissoe WF, Vesey EL, Skvarla JJ (1995) The use of osmium-thiocarbohydrazide for structural stabilization in scanning electron microscopy of pollen. Grana 34: 317-324. doi: 10.1080/00173139509429065

Crane PR (1989) Paleobotanical evidence on the early radiation of nonmagnoliid dicotyledons. Plant Systematics and Evolution 162: 165-191. doi: 10.1007/BF00936916

Cronquist A (1981) An Integrated System of Classification of Flowering Plants. Columbia University Press, New York.

Endlicher S (1840) Genera Plantarum, Secundum Ordines Naturalis Disposita. apud Fr. Beck Universitatis Bipliopolam, Vindobonae.

Erdtman G (1960) The acetolysis method. Svensk Bot. Tidskr. 54: 561-564.

Feng Y-K, Wang X-Q, Pan KY, Hong D-Y (1998) A reevaluation of the systematic positions of the Cercidiphyllaceae and Daphniphyllaceae based on $\mathrm{rbcL}$ gene sequence analysis, with reference to the relationships in the "lower" Hamamelidae. Acta Phytotaxonomica Sinica 36: 411-422.

Ferguson DK (1989) A survey of the Liquidambaroideae (Hamalelidaceae) with a view to elucidating its fossil record. In: Crane PR, Blackmore S (Eds), Evolution, Systematics, and Fossil History of the Hamamelidaceae Volume 1: Introduction and 'Lower' Hamamelidae. Systematics Association Special volume No. 40A. Clarendon Press, Oxford, 273-8.

Fishbein M, Soltis DE (2004) Further resolution of the rapid radiation of Saxifragales (angiosperms, eudicots) supported by mixed-model Bayesian analysis. Systematic Botany 29: 883-891. doi: 10.1600/0363644042450982

Fishbein M, Hibsch-Jetter C, Soltis DE, Hufford L (2001) Phylogeny of Saxifragales (angiosperms, eudicots): analysis of a rapid, ancient radiation. Systematic Biology 50: 817-847. doi: 10.1080/106351501753462821

Hayne FG (1830) Getreue Darstellung und Beschreibung der in der Arzneikunde gebraulichen Gewächse, wie auch solcher, welche mit ihnen verwechselt werden können. 11. Band. Auf Kosten des Verfassers, Berlin.

Hoogland RD, Reveal JL (2005) Index Nominum Familiarum Plantarum Vascularium. The Botanical Review 71: 1-291.

Hoot SB, Magallón S, Crane PR (1999) Phylogeny of basal eudicots based on three molecular datasets: atp B, $r b c \mathrm{~L}$, and $18 \mathrm{~S}$ nuclear ribosomal DNA sequences. Annals of the Missouri Botanical Garden 86: 1-32. doi: 10.2307/2666215

Horaninow P (1843) Tetractys naturae. Typis K. Wienhoeberianis, St. Petersburg.

Hufford LD, Endress PK (1989) The diversity of anther structures and dehiscence patterns among Hamamelididae. Botanical Journal of the Linnean Society 99: 301-346. doi: 10.1111/j.1095-8339.1989.tb00406.x

Ickert-Bond SM, Pigg KB, Wen J (2005) Comparative infructescence morphology in Liquidambar (Altingiaceae) and its evolutionary significance. American Journal of Botany 92: 1234-1255. doi: 10.3732/ajb.92.8.1234

Ickert-Bond SM, Wen J (2006) Phylogeny and biogeography of Altingiaceae: evidence from combined analysis of five non-coding chloroplast regions. Molecular Phylogenetics and Evolution 39: 512-528. doi: 10.1016/j.ympev.2005.12.003 
Ickert-Bond SM, Pigg KB, Wen J (2007) Comparative infructescence morphology in Altingia Noronha and discordance between morphological and molecular phylogenies. American Journal of Botany 94: 1094-1115. doi: 10.3732/ajb.94.7.1094

Jian S, Soltis PS, Gitzendammer MA, Moore MJ, Li R, Hendry TA, Qui Y-L et al. (2008) Resolving an ancient, rapid radiation in Saxifragales. Systematic Biology 57: 38-57. doi: $10.1080 / 10635150801888871$

Kelley RO, Dekker RA, Bluemink JG (1973) Ligand mediated osmium binding: its application in coating biological specimens for SEM. Journal of Ultrastructural Research 45: 254-258. doi: 10.1016/S0022-5320(73)80051-6

Magallón S, Crane PR, Herendeen PS (1999) Phylogenetic pattern, diversity, and diversification of eudicots. Annals of the Missouri Botanical Garden 86: 297-372. doi: 10.2307/2666180

Morley RJ (1999) Origin and evolution of tropical rainforests. Wiley, Manchester.

Nation JL (1983) A new method using hexamehtyldisilazane for preparation of soft tissues for scanning electron microscopy. Stain Technology 58: 347-351.

Noronha F (1790) Verhandelingen van het Koninklijk Bataviaasch Genootschap van Kunsten en Wetenschappen. Egbert Heemen, Batavia.

Pigg KB, Ickert-Bond SM, Wen J (2004) Anatomically preserved Liquidambar (Altingiaceae) from the Middle Miocene of Yakima Canyon, Washingon state, USA, and its biogeographic implications. American Journal of Botany 91: 499-509. doi: 10.3732/ajb.91.3.499

Potter D, Freudenstein J (2005) Phylogenetic Linnaean classification: Taxa should be both ranked and monophyletic. Taxon 54: 1033-1035. doi: 10.2307/25065487

Qiu YL, Chase MW, Hoot SB, Conti E, Crane PR, Sytsma KJ, Parks CR (1998) Phylogenetics of the Hamamelidae and their allies: parsimony analyses of nucleotide sequences of the plastid gene rbcL. International Journal of Plant Sciences 159: 891-905.

Reinsch A (1890) Über die anatomischen Verhältnisse der Hamamelidaceae mit Rücksicht auf ihre systematische Gruppierungen. Botanische Jahrbücher für Systematik 11: 347-395.

Savolainen V, Chase MW, Hoot SB, Morton CM, Soltis DE, Bayer C, Fay MF, De Bruijn AY, Sullivan S, Qiu YL (2000) Phylogenetics of flowering plants based on combined analysis of plastid atp B and rbcL gene sequences. Systematic Biology 49: 306-362. doi: 10.1093/ sysbio/49.2.306

Shi SY, Chang H-T, Chen Y, Qu L, Wen J (1998) Phylogeny of the Hamamelidaceae based on the ITS sequences of nulcear ribosomal DNA. Biochemical Systematics and Ecology 26: 55-69. doi: 10.1016/S0305-1978(97)00075-6

Shi SY, Huang Y, Zhong Y, Du Q, Zhang H, Chang H, Boufford, DE (2001) Phylogeny of the Altingiaceae based on $c p$ DNA matK, PY-IGS and $n r D N A$ ITS sequences. Plant Systematics and Evolution 230: 13-24. doi: 10.1007/s006060170002

Skvarla JJ, Rowley JR, Chissoe WF (1988) Adapatability of scanning electron microscopy to studies of pollen micromorphology. Aliso 12: 119-175.

Soltis DE, Soltis PS, Chase MW, Mort ME, Albach DC, Zanis M, Savolainen V, Hahn WH, Hoot SB, Fay MF, Axtell M, Swensen SM, Prince LM, Kress WJ, Nixon KC, Farris JS (2000) Angiosperm phylogeny inferred from $18 \mathrm{~S} \mathrm{rDNA}, r b c \mathrm{~L}$, and $a t p \mathrm{~B}$ sequences. Botanical Journal of the Linnean Society 133: 381-461. 
Soltis DE, Clayton JW, Davis CC, Gitzendammer MA, Cheek M, Amorim AM, Soltis PS (2007) Monophyly and relationships of the enigmatic family Peridiscaceae. Taxon 56: 65-73.

Soltis DE, Smith SA, Cellinese N, Wurdack KJ, Tank DC, Brockington SF, Refulio-Rodriguez NF, Walker JB, Moore MJ, Carlsward BS, Bell CD, Latvis M, Crawley S, Black C, Diouf D, Xi Z, Rushworth CA, Gitzendanner MA, Sytsma KJ, Qiu Y-L, Hilu KW, Davis CC, Sanderson MJ, Beaman RS, Olmstead RG, Judd WS, Donoghue MJ, Soltis PS (2011) Angiosperm phylogeny: 17 genes, 640 taxa. American Journal of Botany 98: 704-730. doi: 10.3732/ajb.1000404

Soltis DE, Mort ME, Latvis M, Mavrodiev EV, O’Meara BC, Soltis PS, Burleigh JG, Rubio de Casas R (2013) Phylogenetic relationships and character evolution analysis of Saxifragales using a supermatrix approach. American Journal of Botany 100: 916-929. doi: 10.3732/ ajb. 1300044

Takhtajan AL (1997) Diversity and Classification of Flowering Plants. Columbia University Press, New York.

Tardieu-Blot ML (1965) Hamamelidaceae. In: Aubréville A, Tardieu-Blot ML (Eds), Flore du Cambodge du Laos et du Vietnam, Fasc. 4. Paris: Musèum National d'Histoire Naturelle, 75-116.

Tiffney BH (1986) Fruit and seed dispersal and the evolution of the Hamamelidae. Annals of the Missouri Botanical Garden 73: 394-416. doi: 10.2307/2399119

Vink W (1957) Hamamelidaceae. In: van Steenis CGGJ (Ed), Flora Malesiana 5. Nationaal Herbarium Nederland, Universiteit Leiden branch, Leiden, 363-379.

Wen J (1999) Evolution of eastern Asian and eastern North American disjunct distributions in flowering plants. Annual Review of Ecology and Systematics 30: 421-455. doi: 10.1146/ annurev.ecolsys.30.1.421

Wen J (2001) Evolution of eastern Asian-eastern North American biogeographic disjunctions: A few additional issues. International Journal of Plant Sciences 162: S117-122. doi: $10.1086 / 322940$

Williams J (1855) In Balfour J.H. Manual of Botany, ed. 3. J.J. Griffin and Company, Glasgow.

Wu W, Zhou R, Huang Y, Boufford D, Shi S (2010) Molecular evidence for natural intergeneric hybridization between Liquidambar and Altingia. Journal of Plant Research 123: 231-239. doi: 10.1007/s10265-009-0275-z

Yaltrik F, Efe A (2000) Liquidambar orientalis. Curtis's Botanical Magazine 17: 66-71. doi: 10.1111/1467-8748.00249

Zavada MS, Dilcher DL (1986) Comparative pollen morphology and its relationship to phylogeny of pollen in Hamamelidae. Annals of the Missouri Botanical Garden 73: 348-381. doi: $10.2307 / 2399117$

Zhang ZY, Zhang HT, Endress PK (2003) Hamamelidaceae. In: Wu Z-Y, Raven PH, Hong D-Y (Eds), Flora of China, vol. 9. Science Press, Beijing, 18-42. 


\section{Appendix}

\section{Index to Numbered collections:}

Adams, J.W. 177 (styraciflua)

Aksoy 5203 (orientalis), 5204 (orientalis)

Allen, P.H. 11181 (styraciflua), 7265 (styraciflua)

Amador, G. 172 (styraciflua)

Andersen, J.S. 2081 (orientalis)

Araquistain, M. 3538 (styraciflua)

Arp, G.K. 4186 (styraciflua)

Atwood, J.T. 6816 (styraciflua), 9 (styraciflua)

Avendaño, R. 277 (styraciflua)

Bailey, L.H. s.n. (formosana)

Bakhuizen 6372 (excelsa)

Balansa, B. 1156 (formosana)

Balgooy, M.J. v. 2912 (excelsa)

Bartholomew, B. 1758 (acalycina), 1950 (acalycina), 773 (formosana)

Beaman, J.H. 5148 (styraciflua), 5970(styraciflua), 6276(styraciflua)

Bejaud, M. 877 (siamensis)

Berkley, E.E. 966 (styraciflua)

Blanchard, F. s.n. (styraciflua)

Bodinier, E. 1042 (chinensis)

Bogle, A.L. 1561 (orientalis)

Bohs, L. 1798 (styraciflua)

Boratynska, K. 15 (orientalis), 164 (orientalis), et al. 15 (orientalis)

Both, P. 315 (styraciflua)

Botteri 860 (styraciflua)

Boufford, D.E. et al. 37528 (formosana)

Bourgeau, M. 24 (styraciflua)

Boutin, F. 3480 (styraciflua), 3485 (styraciflua), 3494 (styraciflua)

Bracewell, G.L. 42(styraciflua)

Breedlove, D.E. 6886 (styraciflua), 21762 (styraciflua), 34365 (styraciflua)

Breil, D.A. 376 (styraciflua)

Brillet, M. 19 (chinensis)

Burger, W. 26 (styraciflua)

Bush, B.F. 16, 22 (styraciflua), 227 (styraciflua), 2425 (styraciflua), 258 (styraciflua), 3721 (styraciflua)

Calderon, S. 1928 (styraciflua)

Calzada, J.I. 5246 (styraciflua), 5250 (styraciflua), 539 (styraciflua)

Canton Christian College Hebrarium 7700 (formosana)

Carles, W.R. s.n. (formosana)

Carlson, M.C. 608 (styraciflua), 757 (styraciflua) 
Carlson, R. s.n. (styraciflua)

Castillo, C.G. 1350 (styraciflua), 1803 (styraciflua)

Champion 325 (chinensis)

Chandler, A. 2047 (styraciflua)

Chang, H.T. 4701 (acalycina)

Chang, S.Y. 4837 (caudata)

Chen, H. 1272 (gracilipes)

Chen, S.-Q. 14715 (acalycina), 3346 (acalycina)

Cheo, T.Y. 1149 (formosana)

Cheun, Y.R. s.n. (formosana)

Chevalier, A. 2964 (formosana), 37471 (formosana)

Chevez, S.Y. 119 (styraciflua)

Chiao, C.Y. 14238 (formosana)

Chien, S.S. 1019 (formosana)

China Vietnam team 8462 (poilanei)

Ching, R.-C. 1578 (formosana), 2199 (gracilipes), 2244 (chingii), 4823 (formosana), 4823 (formosana), 7937 (chinensis)

Chiu, P.L. 1072 (gracilipes), 1470 (gracilipes), Chiu 961 (acalycina)

Chow, H.-C. 730 (formosana)

Christ, A. s.n. (styraciflua)

Chun, N.K. 42718 (gracilipes), 44321 (obovata)

Chung, H.H. 1006 (formosana), 2132 (formosana), 2901 (formosana), 40 (formosana), 866 (formosana)

Churchill, J.R. s.n. (styraciflua)

Clemens, J. 3388 (siamensis)

Comte, F. 437 (styraciflua)

Conrad, J. 3262 (styraciflua)

Conrad, M.L. 11171 (styraciflua)

Croat, T.B. 25056 (styraciflua)

Cuong, N.M. 93 (formosana)

Curtiss, A.H. 4530 (styraciflua)

Dai, T.L. 103616 (excelsa) after Curtiss

Daniel, T.F. 9628 (styraciflua)

Danish Bot. Trans-Asia Expedition III No. 2081 (orientalis)

Davi, P.H. 13474 (orientalis)

Davidse, G. 34570 (styraciflua)

Davis 40317 (orientalis)

Davis, J. s.n. (styraciflua )

De Voogd 1772 (excelsa)

Delmaree, D. 10971 (styraciflua), 6945 (styraciflua), 14502 (styraciflua), 1661 (styraciflua), 16748 (styraciflua)

Deng, L. 3116 (formosana), 5031 (siamensis), 5438 (gracilipes), 4331 (gracilipes), Dong 4685 (gracilipes) 
Deng, L. 3549 (chingii), 6681 (chingii)

Deramu, R. 1043 (styraciflua)

Dietrich, C. 265 (styraciflua)

Donnat 38180 (formosana)

Dress, D. 4 (styraciflua)

Dumat 414 (excelsa)

Dunn, S.T. 2682 (gracilipes), Dunn s.n. (gracilipes)

Earle, F.S. s.n. (styraciflua)

Eggert, H. s.n. (styraciflua)

Endert, T.H. 1068 (excelsa)

Ewan, J. 17630 (styraciflua)

Exped. of Guizhou Academy of Science 76536 (chingii)

Faircloth, W.R. 3374 (styraciflua), 3709 (styraciflua)

Fan, C.S. 280 (formosana), 75 (formosana)

Fang, W.P. 811 (formosana)

Fassett, N.C. 28302 (styraciflua)

Faurie, A. 279 (formosana)

Faurie, U. 45 (formosana)

Feng, Z. 36293 (gracilipes)

Field Museum 373260 (excelsa)

Fiori, A. 130 (orientalis), 230 (orientalis), 231 (orientalis)

Fischer, G. 20 (styraciflua)

Fleury, F. 30170 (formosana, 37961 (formosana)

Forbes, H.O. 1201 (excelsa)

Forrest, G. 18414 (excelsa), 8763 (excelsa)

Friesner, R.C. 10161 (styraciflua)

Fu, K.-J. 5799 (formosana)

Fujian Forestry Coll. 41 (gracilipes)

Fujian Team 5567 (gracilipes)

Fung, H. 20245 (formosana)

FUS expedition 53260 (chingii)

Gentry, A. 32417 (styraciflua)

Gomez-Pompa 5382 (styraciflua)

Goner 9145 (orientalis)

Gonzalez, L.G. 233(styraciflua)

Greenman, J.M. 4299 (styraciflua)

Gressitt, J.L. 2415 (acalycina)

Grieson, A. 3581 (excelsa)

Griffith 286 (excelsa), 3380 (excelsa)

Grijalva, A. 250 (styraciflua)

Handel- Manzetti, H. 185 (formosana)

Hangzhou Botanial Garden 7303 (caudata)

Harder, D.K. et al. 4180 (formosana) 
Hardial 601 (siamensis)

Harmand 13007 (formosana)

Harper, R.M. 1391 (styraciflua)

Hawkins, T. 176 (styraciflua)

He, G. 4690 (gracilipes), 5479 (gracilipes), 6155 (gracilipes)

He, G.-S. 6179 (formosana)

Henninger, C. s.n. (styraciflua)

Henry, A. 10395 (yunnanensis), 11082 (yunnanensis), 425 (formosana), 5218 (formosana), 52181 (acalycina), 7630 (formosana)

Hernandez, M. 1610 (styraciflua), 448 (styraciflua), 558 (styraciflua), 5630 (styraciflua), 5790 (styraciflua), 6912 (styraciflua), 1499 (styraciflua)

Hers, J. 2264 (formosana), 608 (formosana)

How, F.C. 20124 (formosana), 70369 (obovata)

Howard, R. 627 (styraciflua)

Hu, S.Y. 20953 (formosana), 9984 (gracilipes)

Hu, S.Y. \& K.H. Yung s.n. (formosana)

Huang, Z. 44074 (chingii)

Hubricht, L. 1357 (styraciflua)

Hughes, C.E. 330 (styraciflua)

Hutchens, K. 12 (styraciflua)

Ickert-Bond, S. 1260 (formosana), 1261 (chinensis), 1266 (gracilipes), 1272 (gracilipes), 1274 (chinensis), 1280 (siamensis), 1290 (formosana), 1290, 1291 (formosana), 1291 (formosana), 1296 (poilanei), 1305 (formosana), 1307 (chingii), 1308 (chingii), 1309 (formosana), 1321 (formosana), 1324 (formosana), 1326 (formosana), 1327 (formosana), 1328 (formosana), 1310 (chingii), 1311 (chingii)

Iltis, H.H. 942 (styraciflua)

Institute Bot. Yunnanica s.n. (yunnanensis)

Jayaweera, D.M.A. 1617 (excelsa)

Jensen, M.C. s.n. (styraciflua)

Jones, G.N. 12054 (styraciflua)

Keenan, J. 1407 (excelsa), 1407 (siamensis), 1528 (excelsa), 1940 (excelsa), 3679 (excelsa)

Keng, Y.L. 316 (gracilipes)

Kerr 5110 (siamensis)

Khan 45 (orientalis)

Kin Dao, N. 182 (siamensis)

Kingdon-Ward, F. 20761 (excelsa)

Ko, S.P. 56024 (chinensis), 534488 (chingii)

Koordes 15754 (siamensis)

Kyoto University 41655 (excelsa)

Lammers, T.G. 8304 (styraciflua)

Lau, S. K.1645 (formosana), 4809 (formosana), 25962 (formosana)

Lau, T.W. 98 (gracilipes)

Lau, Y.S. 206 (chinensis) 
Lee, W.N. 6 (styraciflua)

Lei, C.I. 355 (formosana)

Letterman, G. s.n. (styraciflua)

Levine, C.O. 1572 (formosana), 1731 (formosana), 3158 (chinensis), 3302 (formosana)

Li, X. 200701 (gracilipes), 200921 (gracilipes), 202545 (gracilipes)

Li, Z. 1040 (gracilipes), 1162 (gracilipes), 4972 (obovata), 963 (gracilipes)

Li, Z.-X. 3841 (formosana)

Liang 3685 (obovata)

Liang, H.Y. 61283 (chingii), 62594 (obovata), 64371 (obovata), 64734 (obovata), 69714 (chinensis),

Liang, X.-R. 62343 (formosana)

Liebmann, F.M. 3052 (styraciflua)

Liesner, R.L. 26609 (styraciflua)

Lignan Team 12347 (formosana)

Lin, Y.-R. 516 (formosana)

Ling, Y. 2295 (gracilipes), 4522 (chingii)

Liu, L.-H. 542 (acalycina)

Liu, L.L. et al. s.n. (formosana)

Liu, Y. 242 (gracilipes), 90242 (gracilipes)

Lo, L.T. 629 (gracilipes)

Loc, P.K. et al. 1704 (formosana)

Loerzing, J.A. 17368 (excelsa)

Lohman, P. 31 (styraciflua)

Lopez Luna, R. 27 (styraciflua)

M.L. Nie 91192 (acalycina)

M.T. Sin \& W.K. Woo s.n. (gracilipes)

Mackenzie, K.K. 421 (styraciflua)

Manriquez, G. 2339 (styraciflua)

Mao, Z.G. 10231 (gracilipes)

Mao, Z.G. 10237 (caudata)

Marquez, R.W. 997 (styraciflua)

Marshall, E.D. 8711 (styraciflua)

Martinez, S.E. 24118(styraciflua), 3872(styraciflua), 3972 (styraciflua)

Martinez, V.M. 142, 454 (styraciflua)

Matuda, E. 1157 (styraciflua)

May, J.P. 95 (styraciflua)

Maxwell, J.F. s.n (siamensis)

McDonald \& Ismail 4966 (excelsa)

Meijer, Willem s.n. (excelsa)

Mejia, D. 357 (styraciflua), 402 (styraciflua)

Mendez Ton, A. 6087 (styraciflua)

Merrill, E. 1731 (formosana)

Merrill, G.M. 168 (styraciflua), Merrill 61 (styraciflua) 
Metcalf, F.T. \& T.C. Chang 877 (formosana)

Metcalfe, F.P. 9360 (formosana)

Meyer, F.G. 2799 (styraciflua)

Miller, J.S. 9554 (styraciflua)

Moera-Laboch For. Serv, Neth. Ind. 18066 (excelsa)

Molina, R.A. 10807 (styraciflua), 11034 (styraciflua), 11342 (styraciflua), 11454 (styraciflua), 11940 (styraciflua), 12578 (styraciflua), 13755(styraciflua), 20435 (styraciflua), 22235 (styraciflua), 6127 (styraciflua), 7254 (styraciflua), 8430 (styraciflua), 2834 (styraciflua), 983 (styraciflua)

Monro, A.K. 2626 (styraciflua)

Monyrak, M. 10 (siamensis)

Moore, H.E. 1925 (styraciflua), 1995 (styraciflua), 2176 (styraciflua)

Moreno, P.P. 22766 (styraciflua), 25910 (styraciflua)

Muehlenbach, V. 3361 (styraciflua)

Murray, E. 1020 (orientalis)

N.K. Chun 42718 (gracilipes)

Nash, G.V. 860 (styraciflua)

National Geographic Society Hainan Expedition 46 (chingii)

Nee, M. 22484 (styraciflua), 23457 (styraciflua), 26005 (styraciflua), 26198 (styraciflua), 26295 (styraciflua), 26393 (styraciflua), 26885 (styraciflua), 28898 (styraciflua), 29442, 29444 (styraciflua), 29689 (styraciflua), 29700 (styraciflua)

Neil, D.A. s.n. (styraciflua)

Newton, H. 94 (styraciflua)

Ngoc Kinh, H. 169 (chingii)

NGS Hainan Expedition 46 (chingii)

Norris, D.H. 17397(styraciflua)

Norsworthy, J. (styraciflua)

Oersted, A.S. 3050 (styraciflua)

Oldham, R. s.n. (formosana)

Ortega, O.R. 1234 (styraciflua)

Ortega, R. 837 (styraciflua)

Palmer, E.J. 10496 (styraciflua), 14826 (styraciflua), 15227 (styraciflua), 17478 (styraciflua), 17516 (styraciflua), 17884 (styraciflua), 35546 (styraciflua), 44553 (styraciflua), 4805 (styraciflua), 6031 (styraciflua), 8223 (styraciflua), 53823 (styraciflua)

Palmer, E.J. 5235 (styraciflua)

Pammel, L.H. 189 (styraciflua)

Parrott, J.K. 427 (styraciflua)

Pena, M. 1046 (styraciflua)

Perino, C.H. 3262(styraciflua)

Petelot. A. 2332 (chinensis), 5747 (formosana)

Phuong, T. Tien 2539 (formosana)

Phuong 4647 (formosana)

Phuong, V. Xuan 586 (siamensis) 
Pierre, L. s.n. (siamensis)

Ping, K. 1597 (formosana)

Poilane, M. 19024 (yunnanensis), 12844 (poilanei), 1317 (formosana), 13500 (siamensis), 1610 (formosana), 2000 (siamensis), 22153 (siamensis), 3228 (siamensis), 9938 (siamensis), 263 (cambodiana), s.n. (cambodiana)

Polunin, G. 14923 (orientalis)

Prain 769 (excelsa)

Price, S.F. s.n. (styraciflua)

Pringle, C.G. 7754 (styraciflua)

Proctor, G.R. 25212 (styraciflua)

Quaterman, E. 1036 (styraciflua)

Raven, P.H. 19784 (styraciflua), 26323 (styraciflua)

Rechinger, K.H. 8550 (orientalis)

Reed, C.F. 100957 (styraciflua), 10130 (styraciflua), 102408 (styraciflua), 102737 (styraciflua), 10294 (styraciflua), 103177 (styraciflua), 103825 (styraciflua), 10446 (styraciflua), 110706 (styraciflua), 115816 (styraciflua), 116746 (styraciflua), 117576 (styraciflua), 125997 (styraciflua), 126623(styraciflua), 12798(styraciflua), 12807(styraciflua), 129641 (styraciflua), 130382 (styraciflua), 13437 (styraciflua), 136283(styraciflua), 149022 (styraciflua), 22817 (styraciflua), 23414 (styraciflua), 2463 (styraciflua), 2476 (styraciflua), 2533 (styraciflua), 2840 (styraciflua), 29290 (styraciflua), 29353(styraciflua), 3079 (styraciflua), 30915 (styraciflua), 3094 (styraciflua), 31066 (styraciflua), 3213 (styraciflua), 3494 (styraciflua), 35659 (styraciflua), 41634 (styraciflua), 44907(styraciflua), 45066 (styraciflua), 45994 (styraciflua) 4829 (styraciflua), 52801 (styraciflua), 52801 (styraciflua), 52888 (styraciflua), 53398 (styraciflua), 53404 (styraciflua), 7176 (styraciflua), 75623 (styraciflua), 81477 (styraciflua), 82311 (styraciflua), 8946 (styraciflua), 89698 (styraciflua), s.n. (styraciflua)

Regalado, J.C. et al. HLF4449 (siamensis)

Renfrow, J. 16 (styraciflua)

Reyes Rivera, J. 257 (styraciflua)

Rivera, R.J. 907 (styraciflua)

Rock, J.F. 2768 (excelsa), Rock 7174 (excelsa)

Rodriguez, V.J. 1883 (styraciflua), 2840 (styraciflua)

Rossbach, G.B. s.n. (styraciflua)

Rubio, A. 63 (styraciflua)

Rueda, R. 16311 (styraciflua)

Ruth, A. 271 (styraciflua), s.n. (styraciflua)

S.Y. Hu 20064 (formosana)

SABE 1950 (acalycina)

Saito, S. 8371 (formosana),

SAYTBET 45208 (formosana), 45272 (formosana)

Schauer, L.E. s.n. (styraciflua)

Seigler, D. 3601 (styraciflua) 
Seler, E., 3024 (styraciflua)

Seymour, F.C. 205 (styraciflua)

Shekan, J. s.n. (styraciflua)

Shimak, F. s.n. (styraciflua)

Sijam s.n. (styraciflua)

Skutch, A.F. 1739 (styraciflua)

Small, S.K. 292 (styraciflua)

Smith, C.L. 1778 (styraciflua)

Smith, J.D. 1855 (styraciflua)

Smith, T. 3500 (styraciflua)

Smitinand, T. 10848 (excelsa)

Soejarto, D.D. 11399 (formosana), DDS 13 (siamensis)

Solomon, J.C. 2070 (styraciflua, 3719 (styraciflua)

Song, X.H. 1348 (chinensis)

Sousa, M. 3433 (styraciflua)

Sozan, T. 13490 (formosana)

Standley, P.C. 10026 (styraciflua), 13708 (styraciflua), 23747(styraciflua), 56158 (styraciflua), 6497 (styraciflua), 70309 (styraciflua), 887 (styraciflua), Tsoong 256 (multinervis),

Stevens W.D. s.n. (styraciflua), 11510 (styraciflua), 15074 (styraciflua), 17665 (styraciflua), 18095 (styraciflua), 21054 (styraciflua)

Stevens, G.W. 2634 (styraciflua), 3248(styraciflua)

Steward, A.N. 107 (formosana), 1075 (formosana), 29 (formosana), 442 (formosana) Steyermark, J.A. 29732 (styraciflua), 42409 (styraciflua), 49713 (styraciflua), 5172 (styraciflua), 11294 (styraciflua), 11853 (styraciflua), 18919 (styraciflua), 21077 (styraciflua), 6313 (styraciflua), 8662 (styraciflua)

Stone, J. 3096 (styraciflua)

Stratton, R. 585 (styraciflua)

Sun, S.C. 1302 (formosana)

Taam, Y.M. 779 (formosana)

Tan, P.X. 57605 (formosana), 58681 (chingii)

Tang Khoi, N. 420, 421 (formosana)

Tang, C.C. 4655 (formosana)

Tang, H.C. 1473 (formosana)

Tenorio, L.P. 14900 (styraciflua), 5329 (styraciflua)

Thai Van Trung 108 (siamensis)

Thorne, R.F. 40034 (styraciflua)

Tien Ba, N. 110 (siamensis)

Tomlin, S. 176 (styraciflua)

Torres, R. 2541 (styraciflua)

Tsai, H.T. 61528 (excelsa)

Tsang, W.T. 20128 (chinensis), 20927 (formosana), 21514 (gracilipes), 21697 (gracilipes), 21817 (formosana), 22189 (chinensis), 22788 (chinensis), 23613 
(formosana), 25942 (chinensis), 28068 (formosana), 28544 (chinensis), 20927 (formosana), 6412 (styraciflua)

Tsang, W.T. 21697 (gracilipes), 23613 (formosana)

Tsiang, Y. 6677 (siamensis)

Tso 1803 (formosana)

Tso. C.L. 21049 (chinensis)

Tsoong, T.S. 8197 (formosana), 81972 (formosana)

Tsui, T.M. 417 (formosana), 516 (formosana), 660 (formosana)

Tucker, J. M. 1209 (styraciflua)

Tue, H. 523 (siamensis)

Tuerckheim, H. 1804 (styraciflua)

Tutcher, W. J. s.n. (gracilipes)

van Beusekom, C. F. 4102 (siamensis)

van de Koppel 3299 (excelsa)

Vargaz Ruiz, E. 44, 124 (styraciflua)

Vazquez, T.V. 260 (styraciflua), 286 (styraciflua)

Ventura, F.A. 1925 (styraciflua)

Vidal, J.E. 4461 (siamensis)

Vogenberger 8066 (styraciflua)

Wan, X. 153 (gracilipes)

Wang, C. 31824 (formosana), 31828 (chinensis), 35099 (obovata), 35691 (obovata), 35897 (obovata), 36153 (obovata), 36638 (obovata), 37057 (gracilipes), 39334 (chinensis), 44102 (acalycina)

Wang, Y.-J. 56484 (gracilipes)

Warburg, O. 9810 (formosana)

Ward, R.C. s.n. (styraciflua)

Webster, G.L. 17747 (styraciflua)

Wei, Z. 120217 (gracilipes), 120579 (gracilipes)

Williams 306 (styraciflua)

Williams, L. 12190 (styraciflua)

Williams, L.O. 17113 (styraciflua), 28004 (styraciflua), 23388 (styraciflua)

Williston, H. 111 (styraciflua)

Wilson, E.H. 10031 (formosana), 1628 (formosana), 218 (formosana), 513 (acalycina), 795 (formosana), 1639 (formosana), 513 (formosana),

Wu, Z. 85062 (chingii)

Wuyi Shan Team 80-225 (acalycina)

Xiong, B. 20927 (formosana), 2922 (formosana), 5706 (formosana)

Yao, K. 11486 (acalycina)

Yao, K. 11561 (formosana)

Yao, K. 8505 (formosana)

Ye, C.-L. 9689 (formosana)

Yin, H.L. 2031 (formosana)

Yu, B. 103106 (gracilipes) 
Yu. T.T. 229 (formosana)

Yuncker, T.G. 5830 (styraciflua), 8776 (styraciflua)

Zeng, H. 72101 (gracilipes)

Zhang, Z.-Y. 14271 (formosana)

Zhong, Y. 4321 (obovata)

Zola, M.G. 500 (styraciflua), 616 (styraciflua), 651 (styraciflua), 697 (styraciflua), 746 (styraciflua)

Zou, H.-Y. 140 (formosana), 151 (gracilipes), Zou 1984 (acalycina), 307 (chinensis), 761 (chinensis), 762 (gracilipes), 79 (gracilipes)

Zuo, J.B. 356 (acalycina) 